\title{
Binocular Neurons in V1 of Awake Monkeys Are Selective for Absolute, Not Relative, Disparity
}

\author{
B. G. Cumming and A. J. Parker \\ University Laboratory of Physiology, Oxford, OX1 3PT, United Kingdom
}

\begin{abstract}
Most neurophysiological accounts of disparity selectivity in neurons of the primary visual cortex (V1) imply that they are selective for absolute retinal disparities. By contrast, a number of psychophysical observations indicate that relative disparities play a more important role in depth perception. During recordings from disparity selective neurons in area V1 of awake behaving monkeys, we used a disparity feedback loop (Rashbass and Westheimer, 1961) to add controlled amounts of absolute disparity to a display containing both absolute and relative disparities. This manipulation changed the absolute disparity of all the visible features in the display but left unchanged the relative disparities signalled by these features. The addition of absolute disparities produced clear changes in the
\end{abstract}

neural responses to unchanged external stimuli, which were well predicted by the measured change in absolute disparity: in $45 / 53$ cases, the neuron maintained a consistent firing pattern with respect to absolute disparity so that the manipulation created no significant change in the absolute disparity preferred by the neuron. No neuron in V1 maintained a consistent relationship with relative disparity. We conclude that the relative disparity signals used in primate depth perception are constructed outside area V1.

Key words: primary visual cortex; binocular disparity; stereopsis; vergence eye movements; depth perception; receptive field
Since the discovery of disparity selective neurons in the primary visual cortex (V1) three decades ago (Barlow et al., 1967; Nikara et al., 1968), it has been widely assumed that these neurons may form the physiological substrate for stereopsis. To provide a critical evaluation of this hypothesis, it is essential to perform detailed comparisons between the psychophysical properties of stereopsis and the properties of disparity selective neurons.

A striking psychophysical feature of stereopsis is its dependence on relative, rather than absolute, disparity (Westheimer, 1979). The difference between these terms is illustrated in Figure 1. Absolute disparity is simply an angular measure of the difference in the two retinal locations of the projection of a single point (sometimes also called retinal disparity). The relative disparity between two points is also an angular measure, given by the difference between their respective absolute disparities. As Figure 1 shows, changes in the vergence angle of the eyes will cause changes in the absolute disparity of a point, whereas the relative disparity between two points is unaffected.

The geometric fact that relative disparity is independent of eye position may be one reason why it is exploited by the visual system to support so many psychophysical judgements. For example, Westheimer (1979) found that stereoacuity was approximately five times poorer when two isolated targets were presented sequentially as opposed to simultaneously. Simultaneous presentation allows the use of relative disparity signals, whereas sequential presentation forces a reliance on absolute disparity (when performance may be limited by uncertainty about the state of ver-

Received Dec. 23, 1998; revised April 12, 1999; accepted April 14, 1999.

This work was supported by the Wellcome Trust. B.C. is a Royal Society University Research Fellow. We thank Owen Thomas, Andrew Glennerster, Holly Bridge, Jon Dodd, Simon Prince, Greg DeAngelis, and Fred Miles for a critical evaluation of this paper.

Correspondence should be addressed to Dr. Bruce G. Cumming, University Laboratory of Physiology, Parks Road, Oxford, OX1 3PT, UK.

Copyright (C) 1999 Society for Neuroscience $0270-6474 / 99 / 195602-17 \$ 05.00 / 0$ gence). More strikingly, Erkelens and Collewijn (1985) and Regan et al. (1986) found that large changes in the absolute disparity of a wide-field display produced no sensation of motionin-depth. The use of relative rather than absolute disparities in the representation of depth has been compared with the use of contrast rather than luminance in the representation of spatial structure (Rogers and Graham, 1982; Brookes and Stevens, 1989; Howard and Rogers, 1995).

These lines of evidence have resulted in a widely held view that human stereopsis depends primarily on relative disparities. On the other hand, it is widely believed that disparity-selective neurons, at least in primary visual cortex, are selective for absolute, rather than relative, disparities (Joshua and Bishop, 1970; Bishop and Henry, 1971). Nearly all existing physiological data can be explained on the basis of absolute disparities (Barlow et al., 1967; Nikara et al., 1968; Joshua and Bishop, 1970; Bishop and Henry, 1971; Poggio and Fisher, 1977; Poggio and Talbot, 1981; Ohzawa et al., 1990; Ohzawa, 1998), and it is simple to envisage a mechanism that generates selectivity for absolute disparities (by receiving equivalent input from different locations on the two retinae).

Although almost all physiological data in V1 are compatible with a representation of absolute disparity, only two studies (Motter and Poggio, 1984, 1990) have attempted to distinguish between representations based on absolute or relative disparity. They examined this issue by analyzing the effect of errors in convergence (fixation disparities) in awake monkeys. If neurons are selective for absolute disparities, variation in vergence should cause variation in neuronal firing rates. Motter and Poggio (1984) found that vergence errors were large compared with the width of disparity tuning functions. They suggested that the narrowness of observed disparity tuning results from a process that adjusts dynamically for changes in vergence. Motter and Poggio (1990) showed responses from an example cell for which the influence of 


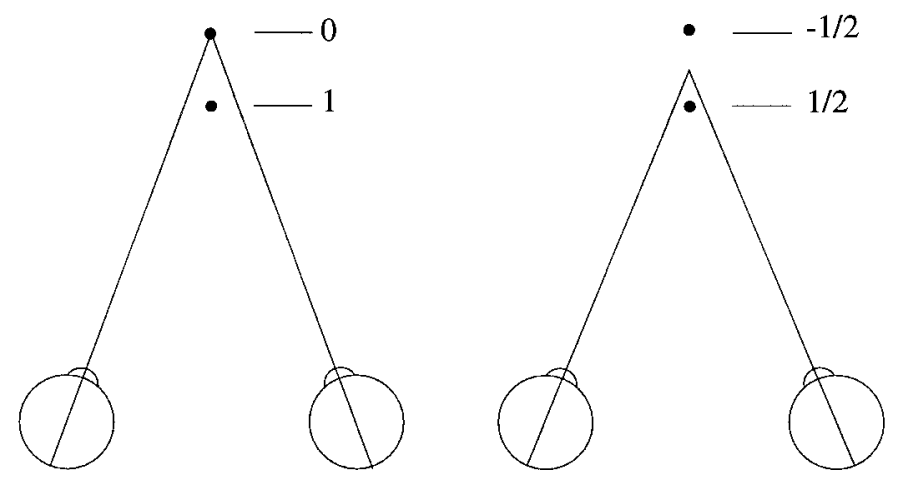

Figure 1. Diagram illustrating relative and absolute disparities of two points in different depth planes. If the vergence angle changes, the absolute disparity associated with each point changes. On the left, the more distant dot is fixated and has an absolute disparity of zero. The near dot then projects to noncorresponding retinal locations and thus has an absolute disparity, arbitrarily assigned 1 unit here. If the depth of fixation changes (right), the absolute disparity of both dots changes (to $\pm 1 / 2$ here). The difference in absolute disparity between the dots is unchanged and is termed their relative disparity.

fixation disparity on firing appears to be smaller than would be predicted on the basis of selectivity for absolute disparity. Both studies concluded that disparity-selective neurons in V1 do not simply encode the absolute disparity of the stimulus within the receptive field. However, this approach relies heavily on the accuracy of binocular eye position recordings (see Discussion).

To summarize, a wealth of psychophysical data indicate that relative, rather than absolute, disparities are important for the perception of stereoscopic depth. The great majority of physiological data in V1 are compatible either with selectivity for absolute disparity or with selectivity for relative disparity. Only two studies have attempted to distinguish these possibilities. Both suggest that disparity-selective neurons in primate V1 neurons may encode relative disparity. Accepting this conclusion would be a major departure from the well developed experimental characterization and models of disparity-selective neurons recorded in the anesthetized visual cortex (Ohzawa et al., 1990; Ohzawa, 1998). We therefore decided to use a disparity feedback loop (Rashbass and Westheimer, 1961) (see Materials and Methods) to apply controlled changes to the absolute disparities of visual stimuli while recording the activity from neurons in V1 of awake primates. This draws a clear distinction between selectivity for absolute disparity or relative disparity.

\section{MATERIALS AND METHODS}

Training. Data were obtained from two adult monkeys (Macaca mulatta), one female $(\mathrm{Rb})$ and one male $(\mathrm{Hg})$. All of the procedures carried out on the animals complied with the U.K. Home Office regulations on animal experimentation.

Animals were trained initially according to the method of Wurtz (1969): pressing a lever illuminated a small spot; after a variable interval the spot dimmed, and the monkeys were rewarded with a drop of water if the lever was released promptly. After this initial training, each monkey was implanted (under general anesthesia) with scleral magnetic search coils (Judge et al., 1980) in both eyes and a head restraining device [modified after Mountcastle et al. (1975)]. After a recovery period of at least $7 \mathrm{~d}$, animals were further trained to maintain fixation during haploscopic presentation of visual stimuli. The positions of both eyes were monitored, and animals earned fluid rewards for keeping the mean conjugate eye position within $0.4^{\circ}$ of the center of the fixation target (a bright dot, $0.2^{\circ}$ in diameter), for periods of $2 \mathrm{sec}$. At this stage, the use of the lever was discontinued, and the only reward criterion was the maintenance of fixation. Finally, the animals were trained to maintain accu- rate convergence when the mirrors of the haploscope were rotated, changing the vergence stimulus. Initially animals did not follow changes in the vergence stimulus accurately; when convergence (to within $0.25^{\circ}$ ) was required to earn rewards, the animals achieved a satisfactory degree of accuracy. After this training, we found that the animals continued to converge accurately, even when this was not required to earn rewards.

Stimulus presentation. Stimuli were generated on a Silicon Graphics Indigo Computer and displayed on two monochrome monitors (Tektronix GMA 201). Gamma correction was applied to produce a linear relationship between luminance and the gray level specified by the computer. The mean luminance was $188 \mathrm{~cd} / \mathrm{m}^{2}$, the maximum contrast was $99 \%$, and the frame rate was $72 \mathrm{~Hz}$. Each eye viewed a separate monochrome monitor through a small circular mirror (18 $\mathrm{mm}$ diameter) placed approximately $2 \mathrm{~cm}$ in front of the eye to form a stereoscope (Wheatstone, 1832). At the viewing distance used $(89 \mathrm{~cm})$, each pixel on the $1280 \times 1024$ display subtended 0.98 arcmin. The "red" video signal was used to control one of the monochrome monitors viewed by the left eye, and the "blue" signal was used to control the other monitor viewed by the right eye. Because the display monitors were monochrome, this allowed the presentation of different black and white images to each eye, while the operator viewed simultaneously an anaglyphic version of the stimulus on a color monitor.

Each mirror of the haploscope was mounted on a galvanometer servomotor (General Scanning G325DP), so that the vergence angle required for bifoveation could be manipulated by rotating the mirrors about a vertical axis. The viewing distance of $89 \mathrm{~cm}$ required convergence of $2.25^{\circ}$ in monkey $\mathrm{Rb}$ and $2.32^{\circ}$ in monkey $\mathrm{Hg}$. Below, vergence angles are described relative to these values: negative angles indicate fixation behind the plane of the monitors and positive angles indicate fixation in front. Similarly, positive disparities are crossed (near), and negative disparities are uncrossed (far). The mirrors took $6 \mathrm{msec}$ to complete a step change in position, much faster than the associated changes in convergence.

Stimuli consisted of bars, sinewave gratings, or random dot patterns, all presented against a mid-gray background. Bar stimuli were used to map out receptive fields. Orientation tuning curves were first constructed with moving bars, then the extent of the minimum response field was delineated with flashing bars at the preferred orientation. Quantitative data on disparity selectivity were then collected with random dot patterns. These were all constructed with equal numbers of white dots and black dots against a gray background. The dot size was usually $0.08^{\circ}$, and the density was $25 \%$. (For a few cells, these parameters were altered to increase response rates). An example of a stereogram is shown in Figure 2.

The random dot stereograms (RDSs) always consisted of a central circular region, whose disparity varied from trial to trial, and a surrounding annulus (usually $0.5^{\circ}$ wide), whose disparity was always zero (hence changes in binocular disparity were not associated with any monocularly detectable changes in the stimulus). The disparities of the center and annulus always remained the same throughout a trial, although a new set of random dots was used on each video frame (i.e., these were dynamic RDSs). The horizontal dimension of the central region was chosen so that at the largest disparity tested the size of the central region covered the minimum response field in both eyes: the size of the central region was thus at least as large as the monocular minimum response field plus the value of the largest disparity to be tested. This precaution was necessary to avoid the possibility that the neuron's receptive field might be incorrectly stimulated with a mixture of the central region and the surround annulus at the largest disparities under test.

During the measurement of a disparity tuning function for a neuron, the disparity was varied from trial to trial in a pseudorandom order, but the order was constrained such that each disparity in the set was shown once before any disparity value was repeated.

A small number of neurons (four) did not respond vigorously to random dot stereograms at any disparity, so they were tested with circular patches of sinusoidal grating stimuli. Spatial and temporal frequency tuning curves were constructed, and the orientation tuning was checked with a stimulus of the optimal spatial and temporal frequency. When disparities were applied, the monocular location of the circular window moved with the grating. This ensured that there was no matching ambiguity in the stimulus, although it had the disadvantage that changes in disparity were associated with detectable changes in the monocular stimulus. When absolute and relative disparities were compared, the stimulus was surrounded by another sinewave grating that remained at zero disparity throughout, providing a good signal for relative disparity 


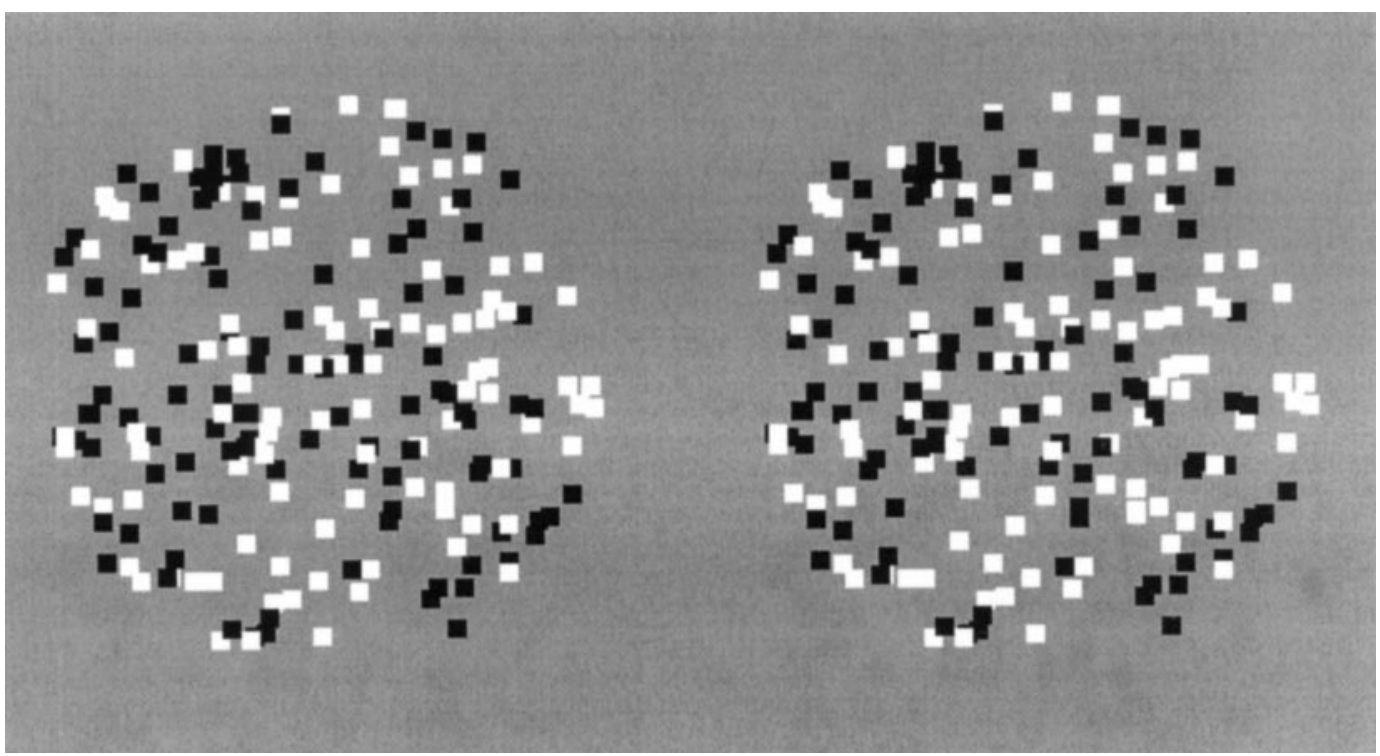

Figure 2. Example of random dot stimulus shown for free fusion.

in the vicinity of the receptive field. The results for these four neurons closely resembled those for the rest of the population.

It would have been possible to manipulate the relative disparity between the foreground and background of the RDS simply by changing the disparity of the background region. However, in such an experiment, the absence of an effect of relative disparity would be hard to interpret. It is always possible that the neurons are sensitive to the disparity relative to some visible feature (such as the fixation marker) other than the one that was manipulated.

It is better to alter the absolute disparity of the entire binocular field while leaving all relative disparities unchanged, including those generated by the fixation marker. This is exactly what happens when a subject converges at a different distance from the fixation marker (Fig. 3). Unfortunately, there is no simple way of exploiting any naturally occurring convergence errors as an experimental manipulation of absolute disparity because (1) if the change in absolute disparity is significant, subjects usually respond with a vergence movement, and (2) if the change in absolute disparity is small, it is hard to measure accurately.

An alternative is to manipulate the absolute disparity of a fixation marker using a feedback loop (Rashbass and Westheimer, 1961). Here, a small disparity is added to the fixation marker (by rotating the mirrors of a haploscope). As subjects attempt to converge on the displaced target, the measured vergence changes are counteracted by additional mirror rotations, clamping the fixation marker at a fixed absolute disparity. This produces a continuous ramp in vergence in both humans (Rashbass and Westheimer, 1961) and monkeys (Cumming and Judge, 1986). The use of a feedback loop allows much greater confidence to be placed in the measured disparity of the fixation marker. If a measured fixation disparity is purely instrumental, no vergence movement will result. Furthermore, because the rate of the vergence movement is proportional to the size of the clamped disparity (Rashbass and Westheimer, 1961; Cumming and Judge, 1986), the vergence movement itself provides an additional check on the absolute disparity of the fixation marker.

Eye movement recording and manipulation. The horizontal and vertical positions of both eyes were monitored by means of a magnetic scleral search coil system (C-N-C Engineering) with the detector time constant set to $0.5 \mathrm{msec}$. The high-frequency noise level was no more than \pm 1 analog-to-digital bit, (0.6 arcmin). However, there also appeared to be some slow drift in the signals over longer periods. The system was calibrated by presenting targets at $2^{\circ}$ either side of straight ahead and adjusting the gain controls until a deflection of $\pm 2^{\circ}$ was recorded for each eye. The signal from one eye in one monkey $(\mathrm{Hg})$ showed a clear asymmetry with respect to straight ahead, so the signal for this eye was calibrated over the actual range of eye movements used. (This asymmetry was most likely caused by the coil in this eye being aligned out of the fronto-parallel plane when the eye was in its primary position, a feature that was evident on visual inspection of the eye.) Note that the calibration for the gain of the eye position signals was performed purely on the basis of conjugate movements; no assumptions about the vergence performance of the animals were made.

The horizontal and vertical positions of both eyes, and the positions of the haploscope mirrors, were digitized and sampled at $587 \mathrm{~Hz}$, allowing vergence angle to be computed on-line. The measured vergence angle was then used to control the position of the haploscope mirrors in a feedback loop. The sequence of events, illustrated by the eye movement records in Figure 4, was as follows: (1) For the first second of a trial, the animal maintained steady fixation at a fixed vergence angle. (2) At $t=1.0$ sec the mirrors were stepped to a new position introducing an absolute disparity of either 0.15 or $0.2^{\circ}$. From this moment, the vergence stimulus was set to be the sum of the measured vergence response and the desired absolute disparity. This led to a smooth vergence movement of nearly constant velocity, as the animal attempted to regain binocular fixation. (In practice, delays in the feedback loop meant that the absolute disparity produced was smaller than the nominal value, but because the positions of both eyes and both mirrors were recorded, it was possible to calculate the real value of the absolute disparity imposed.) (3) At the end of the 2 sec trial, the feedback loop was stopped, and the mirror position was set to a fixed value. This value was set approximately to the mean of the vergence angles reached at the end of such ramps during training. The next trial began with $1 \mathrm{sec}$ of steady fixation at this new vergence angle. (4) Finally, during the next second of this trial, the value of the absolute disparity clamp was of the same magnitude, but of opposite sign, as the preceding trial. Thus the vergence movement was in the opposite direction and returned the vergence angle to its value at the start of the previous trial. (5) The entire sequence was then repeated, so that the value of the absolute disparity clamp alternated between trials. However, the relative disparity of the stimulus was in general different on these sequential trials, because the stimulus order was pseudorandom.

The vergence movement shown in Figure 4 indicates that a disparity has been successfully applied to the fixation marker. Earlier work (Rashbass and Westheimer, 1961; Cumming and Judge, 1986) has shown that the speed of such vergence movements is proportional to the size of the clamped disparity. The fact that the speed of the vergence movement is nearly constant in these records indicates that the disparity of the fixation marker has remained constant during the feedback loop. This provides a useful safeguard against calibration errors: if the speed of the vergence movement is not constant or is much different from the average response to a particular absolute disparity, it suggests that there may be an error in the recorded vergence signal. To give a specific example, if for some reason the recorded vergence is $0.15^{\circ}$ greater than the true vergence position, then an attempt to clamp the disparity at $+0.15^{\circ}$ will result in a true absolute disparity of $+0.3^{\circ}$ and a vergence movement of double the normal velocity, whereas an attempt to clamp the vergence at $-0.15^{\circ}$ will result in no vergence movement at all.

In practice, the average vergence speeds across the entire data set for the two animals studied here were comparable with values reported 
A) Relative: 0, Absolute: 0

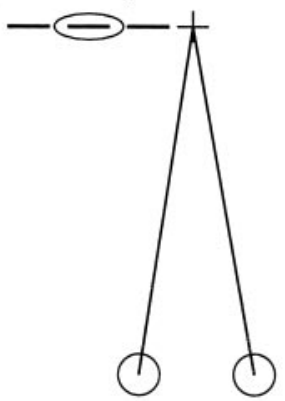

B) Relative: Crossed, Absolute: Crossed

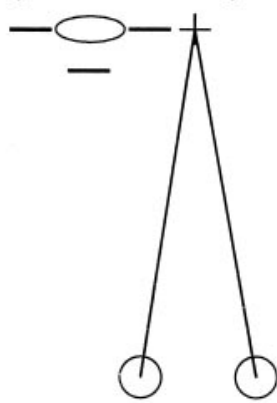

C) Relative: 0, Absolute: Crossed

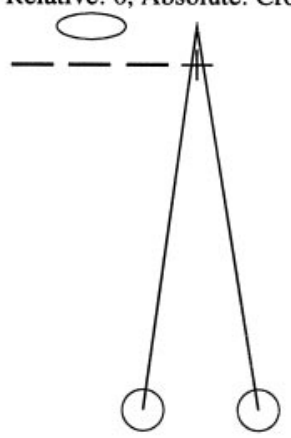

D) Relative: Crossed, Absolute: 0

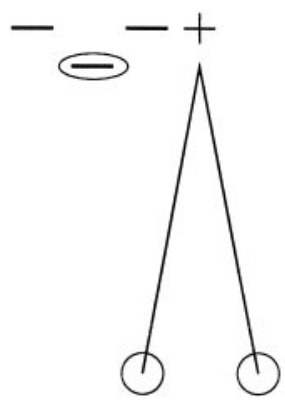

Figure 3. Diagram illustrating the effect of an absolute disparity clamp on two stimulus configurations. Each panel shows a plan view of the two eyes of a subject who is required to fixate the cross while a random dot stereogram (RDS) is presented to the left of the fixation point. The RDS is depicted as the set of thick lines parallel to the interocular axis. Note that the background of the RDS is always at the same location in depth as the binocular fixation marker, whereas the central region of the RDS may be altered in disparity. The ellipse shows the position of an idealized neuronal receptive field, shown at a fixed absolute disparity of $0^{\circ}$. Note that therefore the receptive field is always depicted at the convergence point of the eyes, not at a fixed three-dimensional location relative to the head. Each panel shows a different combination of absolute disparity, either zero or crossed (near), and relative disparity, also either zero or crossed. In $A$ and $C$, the RDS shows a single planar surface at the same depth as the fixation marker, but in $C$ the clamp has placed the central region of the RDS at a crossed absolute disparity. In $B$ and $D$, the central region of the RDS is distinguished by a disparity relative to the surround region, and relative to the fixation marker, but in $D$ the clamp has placed this central region at an absolute disparity of zero. So a neuron selective for zero absolute disparity would respond to configurations $A$ and $D$, whereas a neuron responding to zero disparity relative to the other visible features would respond to configurations $A$ and $C$.

previously in the literature (Rashbass and Westheimer, 1961; Cumming and Judge, 1986). To minimize the effect of any calibration errors, individual trials were excluded from further analysis if the speed of the vergence movement on that trial was $60 \%$ greater or smaller than the mean speed for that clamp size and that animal. Note that this mean speed was calculated over the entire data set, not just for any single day, so the exclusion criterion depends on the absolute error of the imposed clamp. The criterion of $60 \%$ was chosen arbitrarily, mainly because more restrictive criteria caused large numbers of trials to be excluded for a few cells. In practice, removing this criterion altogether had no effect on the overall pattern of results, but we applied it nonetheless because it allowed us to place more confidence in the values of the measured absolute disparities. Figure 5 shows three vergence eye movement traces in response to an absolute disparity clamp of $0.2^{\circ}$. The middle trace illustrates a speed close to the average for this animal and this clamp size, whereas the two outer traces show trials that were excluded from the analysis by the above criterion.

The vergence traces in Figures 4 and 5 show a tendency for the speed of the vergence movement to slow down toward the end of the trial. This effect tended to be larger on those trials in which the initial vergence movement was faster than average. There are several possible reasons for this saturation. First, the vergence stimulus is changing without any change to the accommodative stimulus, so that as the vergence movement proceeds the neural link between vergence and accommodation is likely to defocus the stimulus. Second, the recorded vergence signal may go outside the region in which it is well calibrated. Third, when the vergence angle reached a value that the animal usually associates with the end of the trial, there may be a change in the animal's attention to binocular fixation.

Whatever the reason, the change in the rate of the vergence response indicates that it would be unsafe to rely on the accuracy of the clamp during this period. Consequently, when constructing tuning curves, spikes were only counted from a time $50 \mathrm{msec}$ after the clamp was introduced to a time $750 \mathrm{msec}$ after the clamp was introduced, so that the last $250 \mathrm{msec}$ of each clamp period was excluded. Separate tuning curves were constructed for positive and negative clamps.

Two additional disparity tuning curves were constructed for the periods of steady fixation (one for each static vergence angle). Spikes were counted from a period $50 \mathrm{msec}$ after the first frame of a stimulus appeared until $50 \mathrm{msec}$ after the clamp was applied. For this condition there was no difficulty in using spikes from the last $250 \mathrm{msec}$. Reanalysis of the data using only the first $750 \mathrm{msec}$ of the steady fixation periods did not affect the pattern of results. Thus four disparity tuning curves were constructed for each neuron.

Unit recording and analysis. Once animals were fully trained on binocular fixation, a second operation was performed (under general anesthesia) to implant a recording chamber (Narishige) over the occipital cortex. After a recovery period of at least 1 week, unit recording experiments commenced. Tungsten-in-glass recording electrodes (Merrill and Ainsworth, 1972) were advanced through the dura. The electrode was then usually withdrawn until it sounded as if it were leaving gray matter and allowed to rest for a few minutes before advancing once more and searching for units. The pattern of receptive field locations recorded at different locations from the chamber confirmed that we were recording from primary visual cortex. Neurons with receptive fields too close to the vertical meridian were not included because it was impossible to be sure these were not in V2. Receptive fields were all in the lower right quadrant, at eccentricities from 1 to $4^{\circ}$.

Signals from the electrode were amplified (Bak Electronics) and filtered $(200 \mathrm{~Hz}$ to $5 \mathrm{kHz})$ before being digitized $(32 \mathrm{kHz})$ and stored to disk. The timing of spikes was recorded to the nearest $0.1 \mathrm{msec}$. The storage of spike traces, eye-position signals, and mirror-position signals was performed by the Datawave Discovery System, which also provided a system for on-line classification of spikes. Subsequently, all the spike traces were inspected off-line and reclassified using software developed in our laboratory.

Much of the quantitative analysis relied on fitting curves to the disparity tuning data. Gabor functions were used for this for three reasons. First, many models of disparity selectivity produce tuning functions that are Gabor; second, many of the parameters of the fitted Gabor (such as the phase and spatial frequency) have an intuitive significance; and third, they provided a good fit to the vast majority of the data. The fitting was performed by nonlinear regression (Numerical Algorithms Group). One disadvantage of Gabor functions is that there are frequently multiple local minima, so the resulting fit can be sensitive to the choice of initial parameters. We therefore started the fitting procedure from a large number of different initial conditions and selected the solution with the lowest residual variance. However, in some cases a solution had a low residual, although it was clearly an inadequate description of the data. For example, in some cases the fitted spatial period was smaller than the spacing between the stimuli used, so the fitted curve had peaks and troughs that fell between the real data points. To avoid this, fits with a spatial period smaller than twice the spacing between data samples were not permitted.

\section{RESULTS}

As a whole, 251 neurons were studied (149 from Monkey Rb, 102 from monkey $\mathrm{Hg}$ ). Of these, 53 neurons ( 28 from $\mathrm{Rb}, 25$ from $\mathrm{Hg}$ ) yielded useful quantitative data on the effects of absolute and relative disparities. Most of these cells (51/53) were recorded at eccentricities between 1 and $4^{\circ}$, and the mean receptive field width (estimated by hand plotting, with no corrections for eye movements) was $0.46^{\circ}\left( \pm 0.20^{\circ} \mathrm{SD}\right)$. Cells were classified as simple or complex on the basis of the modulation in their firing to 


\section{vergence stimulus $\quad--\quad$ vergence response - conjugate position}

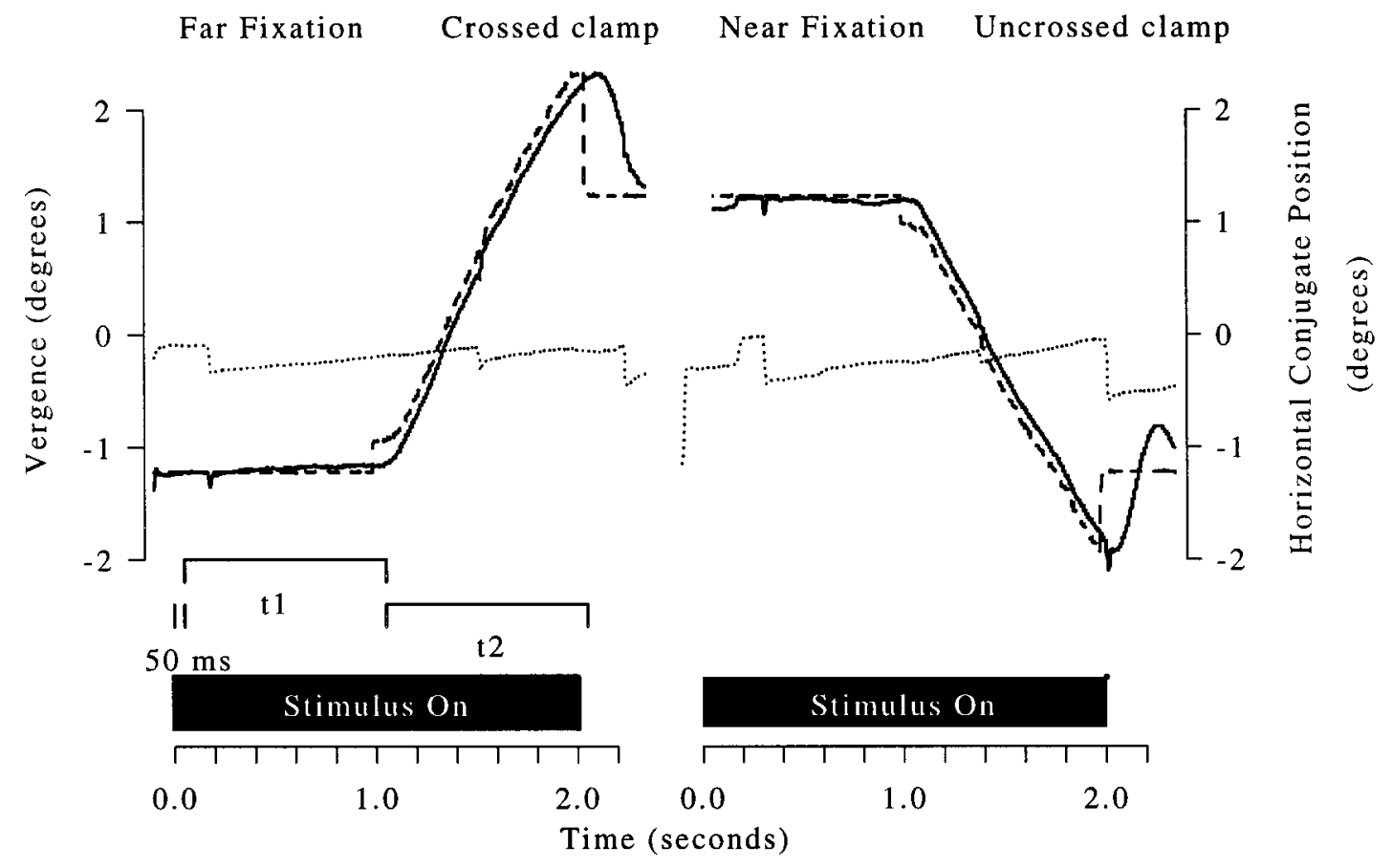

Figure 4. Eye movement records during a period of steady binocular fixation followed by a period in which an additional absolute disparity was imposed by a feedback loop based on measured vergence angle (two sequential trials taken while recording the data shown in Figs. 6-9 below). The dashed line shows the vergence stimulus calculated from the recorded positions of the two haploscope mirror servos; the solid lines show the measured vergence response. For the first $1 \mathrm{sec}$ of each trial, the vergence stimulus is constant. In the left panel, this vergence stimulus is at a relatively diverged position. After $1 \mathrm{sec}$, the mirrors of the haploscope rotate, placing the fixation marker in front of the point of convergence (by $0.2^{\circ}$ in this case). After a reaction time, the animal begins to converge to regain binocular fixation, but the measured vergence position is used to rotate the mirrors further, to maintain the additional absolute disparity of the fixation marker. Thus the disparity of the fixation marker is clamped to the preselected value for a period of 1 sec. Throughout the whole of the $2 \mathrm{sec}$ trial, the same stimulus is presented on the CRT monitors. Note that there is a change in vergence with no systematic change in the conjugate eye position (dotted line, positive values indicate leftward movement, scale at right-hand side of figure). Also, small changes in conjugate eye position are not associated with changes in vergence. Spikes were counted from 50 msec after the beginning of each period, and disparity tuning curves were constructed separately for four conditions: (1) Far Fixation, (2) Crossed clamp, (3) Near Fixation, (4) Uncrossed clamp.

drifting gratings (Skottun et al., 1991), after taking into account eye movements. Of 37 neurons classified in this way, 9 were simple and 28 were complex. (The use of random dot stimuli may have biased the sample toward complex cells.) Of the nine simple cells, five responded to the random dot patterns we used here, and four were tested with grating stimuli. All other neurons were tested with random dot stereograms. All 53 neurons showed some degree of orientation selectivity, and the majority were well tuned. Quantitative data on orientation tuning were stored for 44 units, and this group had a mean orientation bandwidth (halfwidth at half-height) of $34^{\circ}$. The distribution of preferred orientations showed a slight bias toward vertical orientations (34 units had preferred orientations within $45^{\circ}$ of vertical; 19 units had preferred orientations within $45^{\circ}$ of horizontal). No histology is available to confirm the layers from which these recordings were taken, but based on physiological identification of layer IVc, we were able to classify 19 units as infragranular and 29 units as supragranular.

Units that did not fire at a rate greater than $10 \mathrm{spikes} / \mathrm{sec}$ to any stimulus were excluded from the quantitative analysis. We also tended to select strongly disparity-selective neurons for this study. If the modulation attributable to disparity was weak, albeit statistically significant, the neuron was usually excluded. Finally, a one-way ANOVA was performed on each of the four disparity- tuning functions constructed for each cell, and neurons were included only if they showed a significant effect of disparity $(p<$ $0.05)$ independently in all four cases. In practice, our informal criteria applied at the time of recording usually ensured that this was true: only three units were excluded from the study by this final criterion.

\section{Effects of adding absolute disparities}

The effect of an absolute disparity clamp on vergence eye movements has been described in Materials and Methods. A clamp adds a fixed absolute disparity to the entire display (including the fixation marker and any visible part of the CRT monitors themselves), and leaves the entire pattern of relative disparities between all visible elements unchanged. The effects on neural activity are illustrated for one neuron in Figures 6-9. Figure 6 shows the response to a stimulus at the preferred disparity $\left(0.2^{\circ}\right.$, average of five trials). All of the relative disparities are unchanged during the $2 \mathrm{sec}$ trial because the same stimulus is presented on the display screens throughout. Nonetheless, there is a dramatic change in firing rate when the movement of the mirrors adds an additional absolute disparity to the retinal stimulus. Changes in absolute disparity change the mean firing rate (calculated over the first $750 \mathrm{msec}$; see Materials and Methods) to a constant 


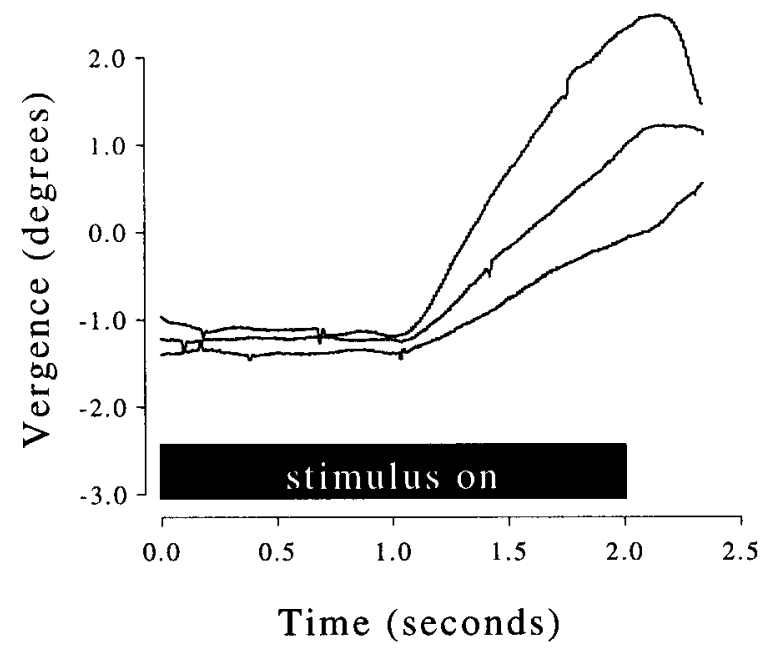

Figure 5. Vergence eye movements from three individual trials with a nominal disparity clamp of $0.2^{\circ}$. The top and bottom traces show examples of trials in which the velocity of the vergence movement caused the trial to be excluded from the analysis. The central trace shows a trial in which the vergence velocity was near the mean for this animal and this clamp size. In the bottom trial, the animal appears to be underconverged during the fixation period, and this is followed by a relatively slow convergence ramp. This is to be expected if the measured underconvergence is an instrumental artifact. If convergence is underestimated, the real clamp applied will be smaller than that measured. Similarly, the top trace shows initial overconvergence and a relatively fast ramp.

external stimulus. This indicates that the neuron is not simply selective for relative disparities.

Figure 7 shows how additional absolute disparities changed the neuron's responses to a range of relative disparities. The left panel shows disparity tuning plotted as a function of relative disparity (relative to the fixation marker). Thus, identical stimuli on the CRT monitors are plotted in the same positions on the abscissa. The right-hand graph plots the same responses as a function of absolute disparity. For each stimulus, the mean of the measured disparity clamp was used to calculate the additional absolute disparity imposed on the stimulus by the clamp procedure. These additional absolute disparities were added to the relative disparities already present on the CRT monitors to calculate the absolute disparity of the stimulus on the retina. The right-hand panel of Figure 7 shows that this neuron gives a consistent pattern of behavior with respect to absolute disparity rather than relative disparity. The magnitude of the change in firing rate to a fixed physical stimulus is well predicted by the measured absolute disparity of the clamp. Figure 8 shows the response of four other neurons, two from each monkey, plotted as a function of absolute disparity. All four cases show a consistent relationship with absolute disparity.

\section{Are the responses of the population better described by absolute} or relative disparity?

The fact that additional absolute disparities alter the responses to relative disparity can be confirmed by a two-way ANOVA: for $51 / 53$ cells, the interaction between relative disparity and clamp condition was significant $(p<0.05)$. One might expect that the same approach could be used to detect any interaction between absolute disparity tuning and clamp condition. Unfortunately, an ANOVA on the absolute disparity responses cannot be performed because the absolute disparities of the stimuli were not

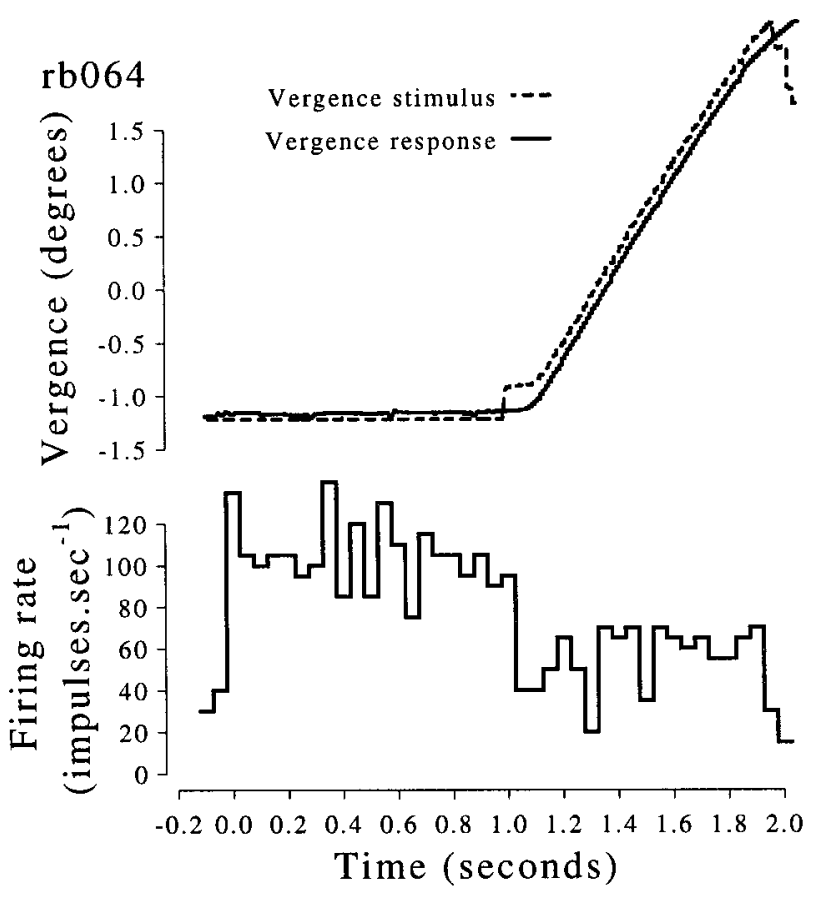

Figure 6. Effects of the feedback loop on activity of one disparityselective neuron. The stimulus throughout was presented at a disparity of $0.2^{\circ}$ relative to the fixation marker, the preferred disparity when tested during steady fixation. When the absolute disparity of the fixation marker is changed (by $0.172^{\circ}$ created by setting a target disparity clamp of $0.2^{\circ}$; see Materials and Methods and Fig. 7), so that the absolute disparity of the stimulus is $0.372^{\circ}$, the firing rate drops immediately, although the relative disparity is unchanged. Average of five trials.

identical for the two clamp conditions. This occurred because the measured value of the clamp differed slightly from the value designated in the feedback loop (see Materials and Methods). Thus, in Figure 7 the disparity tuning curve was measured at intervals of $0.2^{\circ}$. The value of the absolute disparity clamp was set to $\pm 0.2^{\circ}$, but the measured clamp size was actually $\pm 0.17^{\circ}$. Thus the data points from the two clamp conditions in Figure 7 are at slightly different positions along the abscissa.

Hence, we require an alternative way of testing whether the responses to absolute disparity are consistent. Ideally, the same test should also be applicable to the responses to relative disparity. We approached this by fitting Gabor functions to the disparity tuning data, as illustrated in Figure 9. A single curve was fitted to the combined data sets from the two clamp conditions. This procedure was applied separately to tuning curves expressed in terms of relative disparity and absolute disparity. If the neuron were to maintain a consistent response to relative disparity, then the residual variance around a single curve fitted to the relative disparities should be smaller than for a curve fitted to absolute disparities. On the other hand, if the neuron were to behave consistently with respect to absolute disparity, then the reverse should be true. Figure 10 plots the residual variance around a single Gabor fitted to the mean firing rates for each cell. This Gabor was fitted to the data expressed in terms of either relative disparity or absolute disparity. For all 53 cells, the fit to the absolute disparity data had a lower residual variance than the fit to the relative disparity data.

This analysis indicates that for all 53 cells the responses bear a 


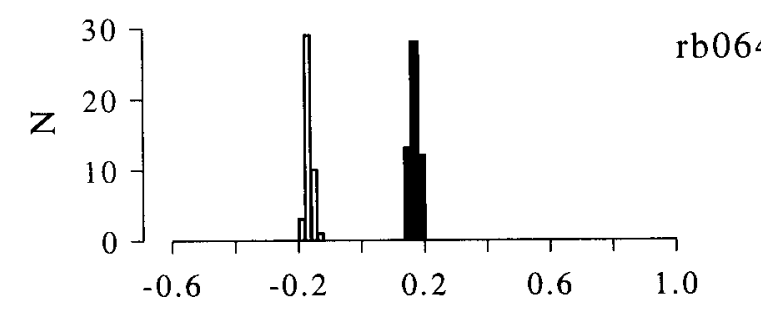

Added absolute disparity $+0.172^{\circ}$
Added absolute disparity $-0.167^{\circ} \mathrm{O}$

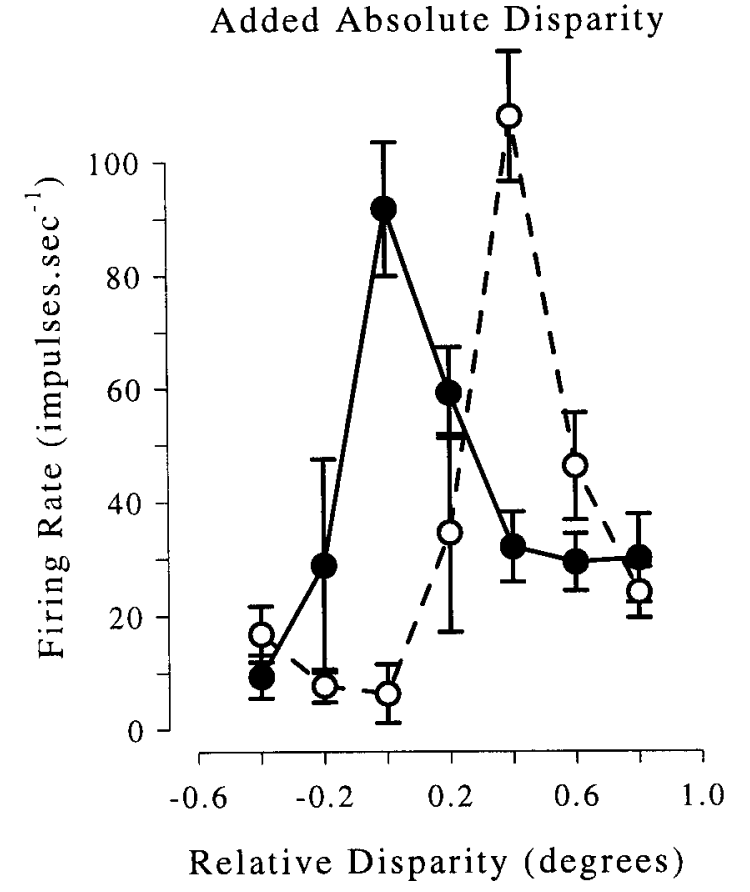

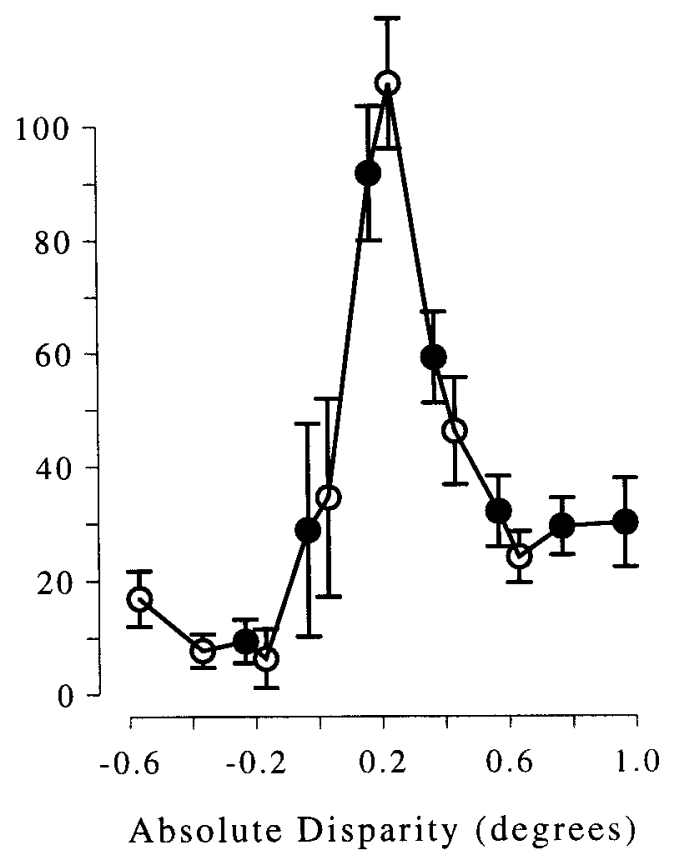

Figure 7. Disparity tuning curves for the cell illustrated in Figure 6. The data were collected during the imposition of two different, additional absolute disparities. The left panel shows the data plotted in terms of relative disparity (the disparity of the stimulus within the receptive field relative to the fixation marker). A frequency histogram is also shown for the measured absolute disparity of the fixation marker during each clamp. The right panel shows the neural data replotted in terms of absolute disparity (the disparity of the stimulus with respect to retinal landmarks such as the fovea). Each tuning curve from the left panel has simply been moved horizontally by the mean measured absolute disparity value for the respective clamp condition.

closer relationship to the absolute disparity of the stimulus within the receptive field than to the relative disparities between the receptive field and the rest of the visible scene.

\section{How accurately can the tuning curves be described by} absolute disparity?

The previous analysis does not by itself establish that the responses of V1 cortical neurons are accurately described in terms of absolute disparity. It could be that the responses are intermediate between the two extremes but lie closer to absolute than to relative disparities. To evaluate this possibility, an additional parameter was introduced to the fits. This allowed separate Gabor functions to be fitted to the tuning data for the two clamp conditions. All the parameters of the two Gabor fits were identical, except for a horizontal displacement. This fitting procedure was again applied to data expressed in terms of both relative and absolute disparity. Examples of these Gabor + Shift fits are shown in Figure 9.

For a neuron that maintains a consistent relationship to absolute disparity, the value of this fitted shift should be small when the data are expressed in terms of absolute disparity. When the same data are expressed in terms of relative disparity, the value of the shift should be equal to the magnitude of the absolute dis- parity difference between the two conditions. On the other hand, for a neuron that is selective for relative disparity, the opposite pattern should hold: the shift should be small when the data are expressed in terms of relative disparity and equal to the change in absolute disparity when the data are expressed in terms of absolute disparity.

The statistical significance of these shifts can be assessed with a sequential $F$ test (Draper and Smith, 1966), in which the variance accounted for by adding the shift term is divided by the residual variance around the fit that includes the shift. This test was applied separately to each unit, and in every case there was a significant shift $(p<0.05)$ in the tuning to relative disparities under the two different clamp conditions. This means that adding an absolute disparity always significantly alters the responses of V1 neurons to relative disparity stimuli. Conversely, when the fitting procedure was applied to absolute disparities, a significant shift was present in only $8 / 53$ cells. For the majority of cells $(85 \%)$, absolute disparity by itself gave a completely satisfactory description of the disparity tuning functions under the two clamp conditions.

The sizes of the fitted shifts in the tuning functions are highly informative. They are shown in Figure $11 A$, where a frequency histogram for the 53 cells is shown. This is a unimodal distribu- 

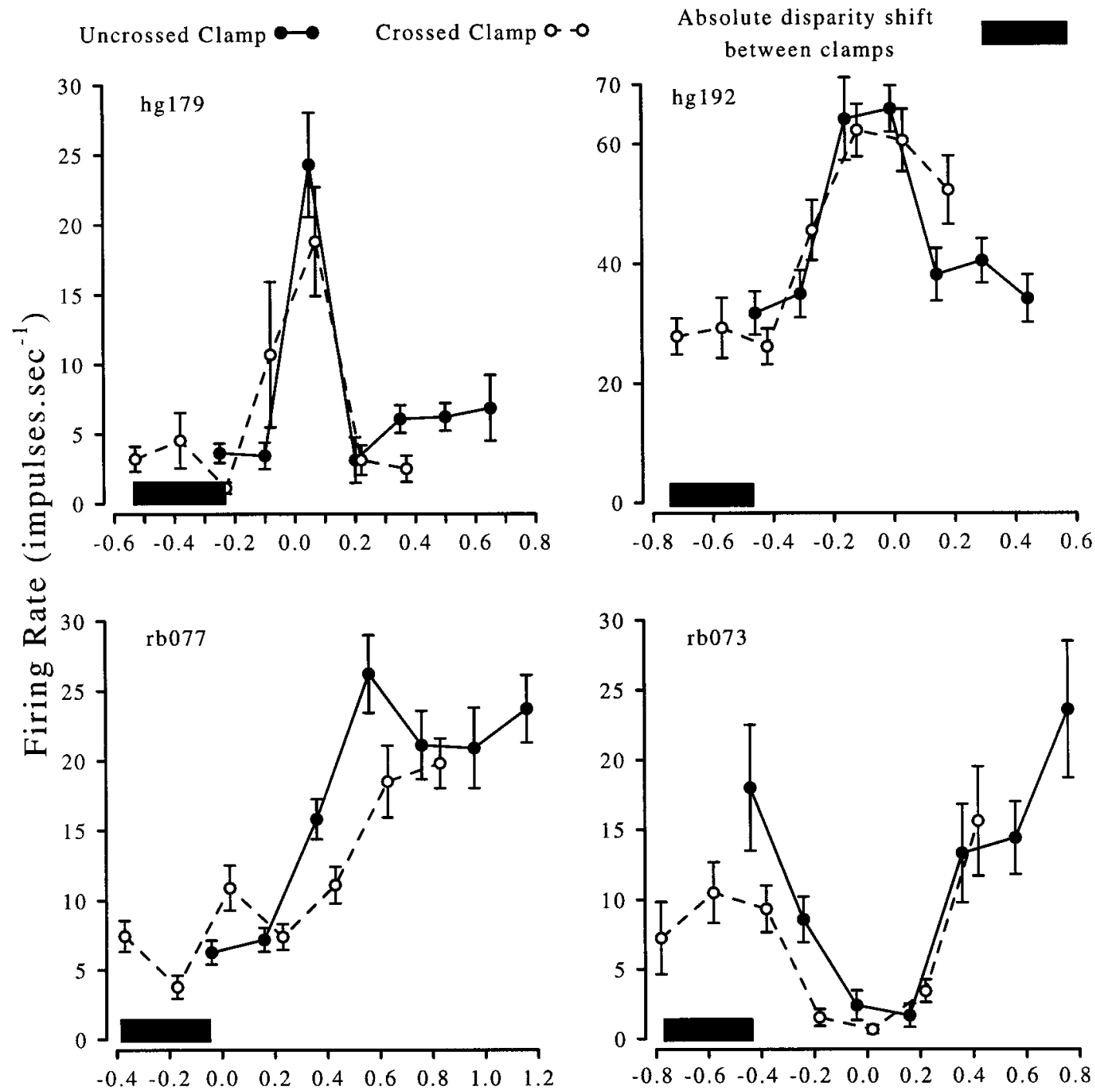

Absolute Disparity (degrees)

Figure 8. Disparity tuning functions for four units, plotted as a function of absolute disparity. Two units from each monkey are shown. Various tuning types [as identified by Poggio and Fisher (1977); Poggio (1995)] are represented: TE/T0 cells (top row, showing a maximal response to disparities near zero), a Near cell (bottom left, showing a stronger response to near disparities than to far disparities), and a TI cell (bottom right, suppressed by near-zero disparities). The solid bar in each graph indicates the size of the absolute disparity shift produced by the clamp. Shifting the solid symbols by this distance to the left would align the responses in terms of relative disparity. In all cases, there is a consistent relationship to absolute, not relative, disparity.

tion with a mean $\left(-0.011^{\circ}\right)$ not significantly different from zero $(t$ test, $p>0.05)$. The clamp procedure induced a difference in the added absolute disparities of between 0.2 and $0.4^{\circ}$ for the two clamp conditions, depending on the experimental conditions that applied for each neuronal recording. It is evident that there is no tendency for the shifts to cluster in this region along the abscissa.

To account for the differences in the clamp size between experiments, we calculated a scaled shift for each cell. This is the shift fitted to the absolute disparity data divided by the difference in the additional absolute disparity created by the two clamp conditions. For an ideal neuron selective only for absolute disparity, this scaled shift should be 0.0 (no shift in tuning expressed in absolute disparity). For an ideal neuron selective only for relative disparity, the scaled shift should be 1.0; that is, the tuning curves should be separated by an amount equal to the added absolute disparity. The frequency histogram for the 53 cells is shown in Figure $11 C$. Again, this is a unimodal distribution with a mean $(-0.032 \pm 0.184 \mathrm{SD})$ not significantly different from zero $(t$ test, $p>0.05)$. Note that values of exactly zero indicate that the fitted shift in tuning exactly matched the measured size of the absolute disparity clamp.

It appears that disparity-selective neurons in V1 represent a homogeneous group that are selective for absolute, not relative, disparity. There are neurons that show a statistically significant shift, but as Figure $11 C$ shows, they are found at both tails of the unimodal distribution. If these neurons with significant shifts represented a subpopulation with a degree of selectivity for relative disparity, then they should fall on the right-hand side of the distribution (nearer to a scaled shift of 1.0, which corresponds to selectivity for relative disparity). In fact, there are more negative shifts (six) than positive shifts (two), arguing against any representation of relative disparity in V1. 

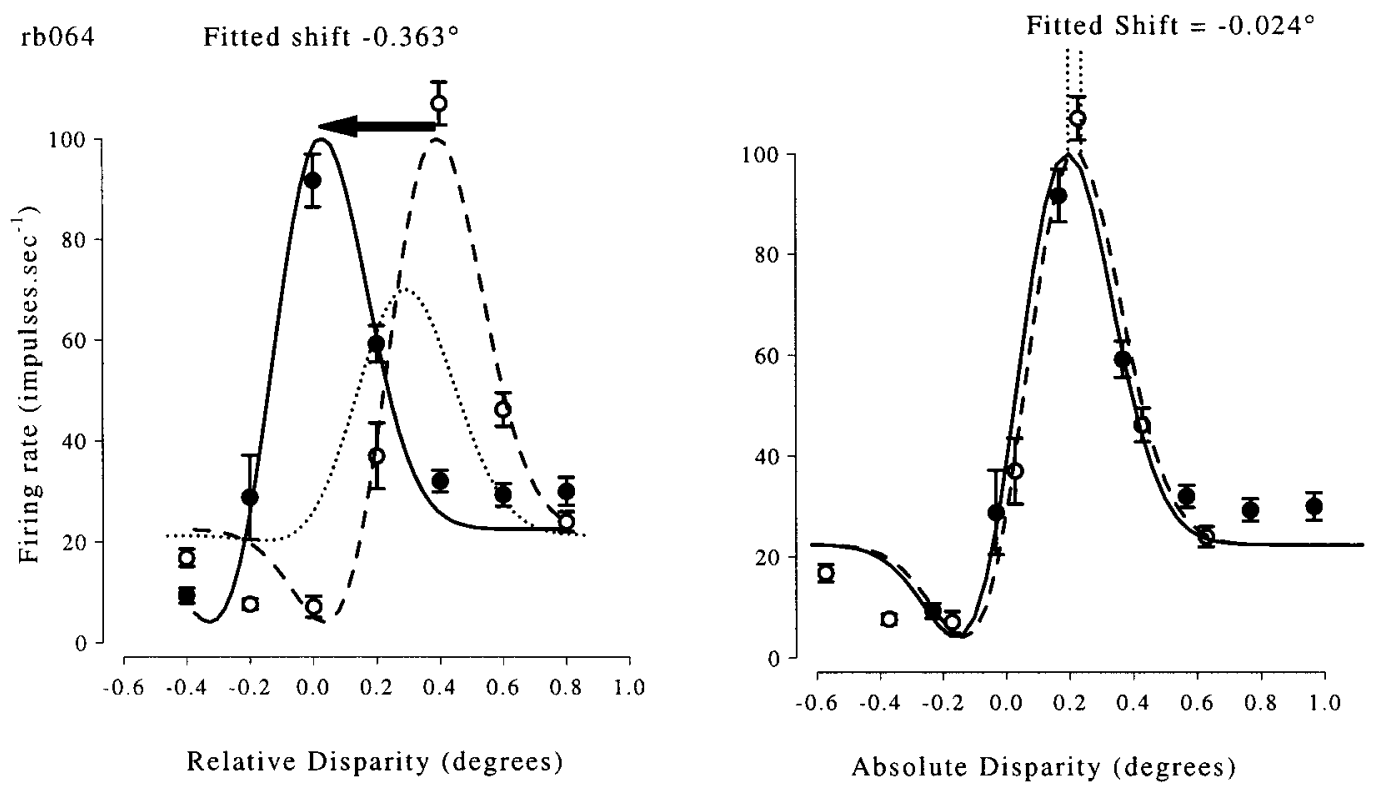

Figure 9. Gabor functions fitted to the tuning data shown in Figure 7. To begin, two Gabor functions are fitted, one to each clamp condition; that is, one Gabor for the additional absolute disparity with a positive value and another Gabor for the one with a negative value. The parameters of the two Gabor functions are identical, except for a horizontal translation. The magnitude of the horizontal translation then gives a measure of how consistently the neuron relates to a given experimental variable. Clearly, the relationship to relative disparity (left) is not consistent, because there is a substantial horizontal shift in the curve when the absolute disparity is changed. Furthermore, the size of the horizontal shift measured from the fitted curves $\left(-0.363^{\circ}\right)$ is very similar to the measured difference in additional absolute disparity between the two conditions $\left(-0.339^{\circ}\right)$. Consequently, when the data are expressed in terms of absolute disparity and the same comparisons are made, the fitted shift is very small $\left(0.024^{\circ}\right)$. The significance of these shifts can be assessed by comparing the goodness of fit of the linked pair of Gabor functions with a single Gabor that attempts to describe the combined data set. Adding the horizontal shift increases the number of parameters by one. On the left, the single Gabor is clearly a poor fit (dotted line); the addition of the horizontal shift to create a pair of linked Gabor functions improves the fit $\left(F_{(1.86)}=408, p<0.00001\right)$. On the right, using the pair of linked Gabor functions does not significantly improve the fit $\left(F_{(1,86)}=3.0, p>0.05\right)$ compared with a single Gabor. (In fact, the fit with a single Gabor is so similar to the two illustrated curves that it is not shown separately.) It can be concluded that the firing rate bears a consistent relationship to absolute disparity regardless of the added disparity clamp.

\section{How good is the model used to assess shifts in the disparity tuning functions?}

So far, the analysis has assumed that the disparity tuning data can be well described by the Gabor+Shift curves. This assumes that the shape of the tuning curve remains constant when the absolute disparity of the display is altered, allowing only for changes in the neurons' preferred disparity. To assess the possibility that there were other changes produced by the absolute disparity clamps, we also fitted two completely independent Gabor functions to the tuning curves for the two conditions and performed a sequential $F$ test to determine whether there was a significant reduction in the variance around the fit. Two independent Gabor fits were a significant improvement on the linked Gabor+Shift fit in only 10/53 cases. Indeed, when two independent Gabors with a single Gabor (no shift) fitted to the absolute disparity tuning were compared, only 14/53 neurons showed a significant improvement in the fit. Thus the responses of 39/53 neurons (74\%) are well described solely by their response to the absolute disparity of the stimulus within the receptive field.

Even for the cells that appear to deviate from a consistent relationship to absolute disparity, the magnitude of the deviation was generally small. The cell shown in Figure 9 is 1 of the 10 cells that shows a significant improvement when a fit is performed by two independent Gabors, yet it is clear that the data are quite well described by a single Gabor. To quantify the size of the improvement produced by two independent Gabor fits, the fraction of the variance that was explained by the two fits was calculated. On average, $88 \%$ of the variance was accounted for by the
Gabor+Shift fit, and this figure was $96 \%$ for the fit with two independent Gabors. (This also confirms that the Gabor functions provided good fits to the tuning functions.) These deviations therefore do not raise any substantial difficulties for the main conclusion that disparity-selective neurons in primate V1 are selective for absolute, not relative, disparities. Possible reasons why the responses of a minority of neurons appear not to be simply described by absolute disparity will be considered in the next section.

\section{Effects of changes in vergence alone}

The vergence movements produced by absolute disparity clamps serve as a useful tool for manipulating absolute disparities independently of relative disparities. However, the changes in vergence angle also raise a potential complicating factor: changes in vergence angle by themselves may have a significant effect on disparity tuning. An interaction of this type has been reported (Trotter et al., 1992, 1997). It was important to determine whether similar interactions were present in this study. For this reason, data were collected at two different vergence angles, covering approximately the range of movement produced by the clamps (see Materials and Methods) and interleaved with the clamp data.

It should be pointed out at once that it will be impossible to make a direct comparison between our results and those of Trotter et al. $(1992,1997)$ because there are several critical differences in the experimental conditions. Notably, Trotter et al. $(1992,1997)$ changed vergence by changing the physical viewing distance over a wide range (from 80 to $20 \mathrm{~cm}$, corresponding to 


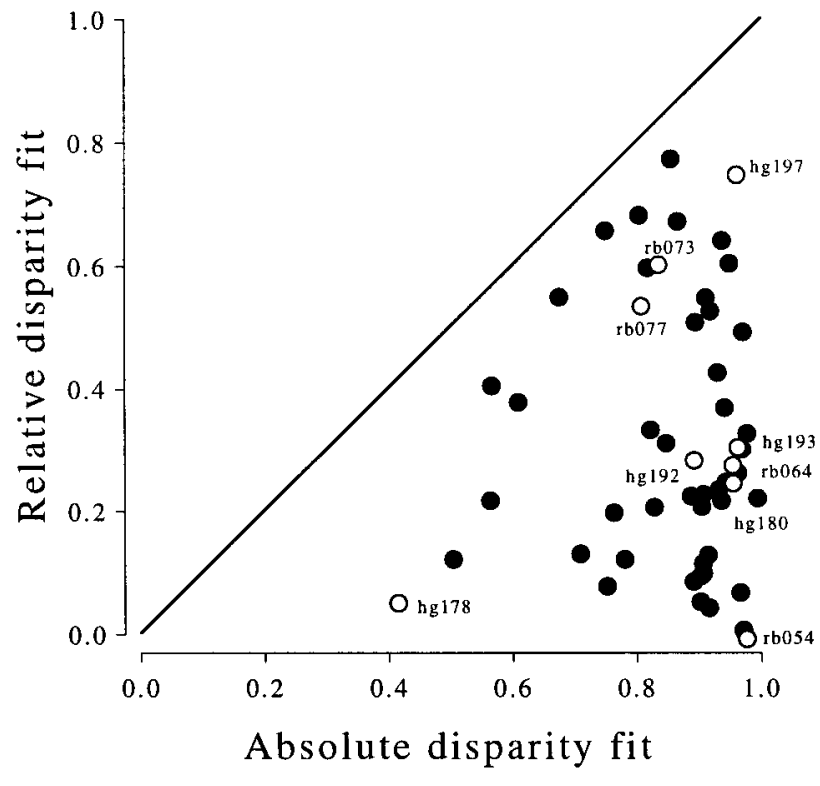

Figure 10. Comparison of goodness of fit of a single Gabor function fitted to relative disparity or absolute disparity tuning functions. The fraction of the total variance accounted for by a single Gabor fit to the mean firing rates as a function of disparity was calculated. Note that both fits have the same number of free parameters. Cells shown with open symbols are those for which tuning curves are shown elsewhere. For all 53 cells, fitting a Gabor to the absolute disparity data produces a better description than a fit to the relative disparity data: all cells maintained a more consistent relationship to absolute disparity than to relative disparity. For points far away from the identity line (solid line), this difference was large. Points might fall close to the identity line for several reasons. (1) If the shift in absolute disparity is small compared with the disparity bandwidth of the cell (see rb073, Fig. 8, bottom right), then both fits will be good. (2) For Near/Far cells, if only one or two data points fall on the sharply changing region of the tuning function (the only region affected by the clamp), neither fit need be very poor (see rb077, Fig. 8; hg197, Fig. 16). (3) If there is a shift in preferred relative disparity, but this shift is not equal to the absolute disparity measured from eye and mirror position records, then the fit to both relative and absolute disparities will be imperfect (rb073 in Fig. 8 is an example where the shift in the tuning curves appears slightly less than the measured shift). (4) If a single Gabor function is a poor description of the disparity tuning curve, both fits will be poor, typically because either the neurons are only weakly modulated by disparity or there is a change in the shape of tuning curve between the two clamp conditions (hg178, Fig. 17).

vergence angles of $2-10^{\circ}$ for a monkey with an interocular separation of $3.5 \mathrm{~cm}$ ). This was larger than the range of $1.0-3.5^{\circ}$ studied here with the mirror haploscope. For this reason, any interactions between disparity tuning and vergence may be smaller in our results. Also, in the work presented here, the positions of both eyes were monitored, and accurate vergence was required behaviorally of the animal, whereas vergence was not monitored in the studies of Trotter et al. (1992, 1997).

The main aim of the following analysis is to establish the extent to which any vergence-related changes in the disparity tuning of cortical neurons can be identified within the present data set. As will become apparent, small but significant changes are identifiable in some of our data, so the second aim is to examine the underlying causes of these changes. In particular we examine the importance of fixation disparity during static vergence and the significance of careful measurement of the receptive field characteristics before examination of the neuron under different vergence states. For our data set, the results can be reconciled with
A)

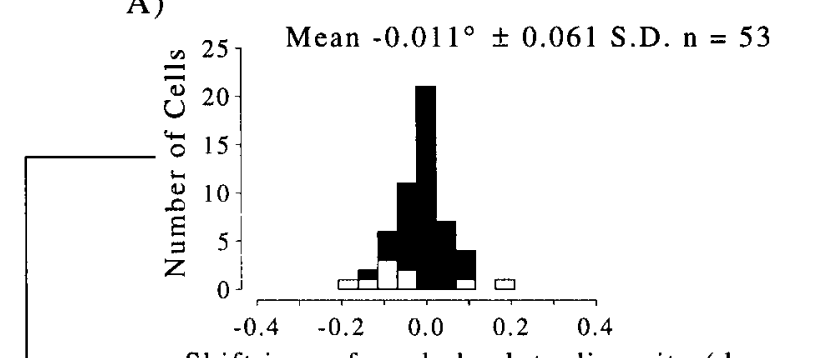

Shift in preferred absolute disparity (degrees)

B)

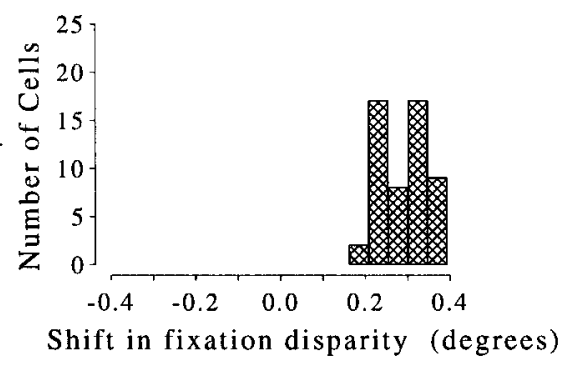

C)

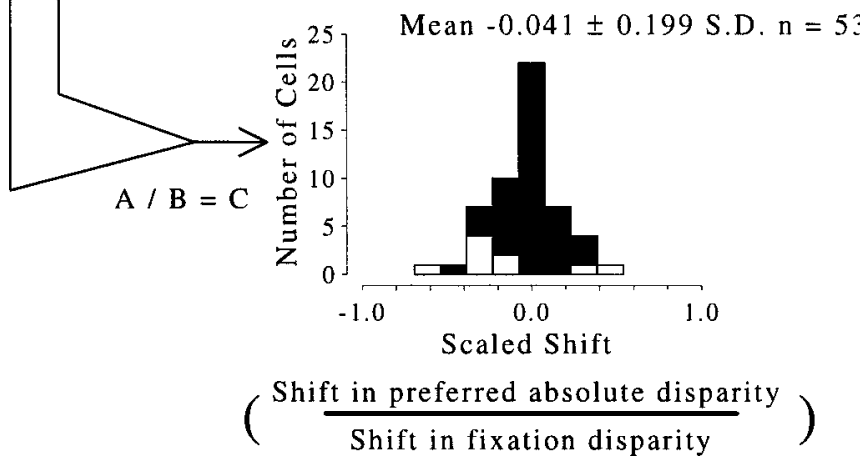

Figure 11. Frequency histograms showing the distribution of $(A)$ shifts in preferred absolute disparity, $(B)$ shifts in measured absolute disparity of the fixation marker (fixation disparity), and $(C)$ the ratio of these measures, termed scaled shift. For each neuron, the tuning to absolute disparities was fitted with a pair of Gabors differing only in their horizontal position, and the shift between the two Gabors was calculated (the shift in absolute disparity preference). For the same set of trials, the actual size of the absolute disparity clamp was calculated from records of mirror and eye position, and the difference between the two clamp conditions was calculated (shift in fixation disparity). The ratio of these measures (scaled shift) gives a measure of the extent to which disparity preference was influenced by the clamp. Values of zero correspond to a consistent relationship with absolute disparity. If a neuron maintained a consistent relationship to relative disparity, then the tuning when plotted in terms of absolute disparity should shift by a disparity exactly equal to the change in absolute disparity, giving a scaled shift of 1.0. The eight units shown in white had significant alterations in their selectivity for absolute disparity under the two clamp conditions. Note that values of 0 do not arise simply because there is no measurable change in disparity selectivity; rather, they arise when the change in response to a stimulus of fixed relative disparity is exactly explained by the measured change in absolute disparity produced by the clamp.

the conclusions from the disparity-clamp data, which point to absolute disparity being the critical parameter for disparityselective neurons in V1.

\section{Disparity selectivity at different vergence angles}

Some of the most extreme changes found by Trotter et al. (1992, 1997) at different viewing distances involve what is essentially the 


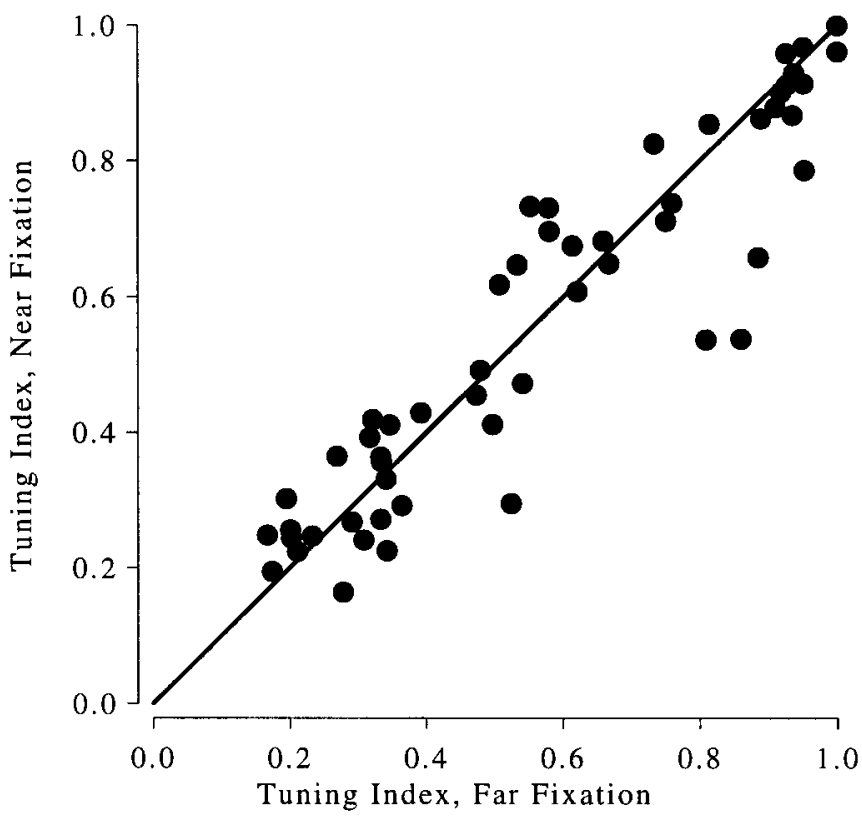

Figure 12. Effects of vergence changes on disparity tuning index for the 53 neurons studied here. The tuning index is similar at both fixation distances. The solid line is the identity line.

complete loss of disparity tuning at one viewing distance (typically a near viewing distance of $20 \mathrm{~cm}$ ). For our dataset, one simple comparison of disparity tuning at the two vergence angles is presented in Figure 12, which is a scatterplot of disparity tuning index, measured at two vergence angles, where:

disparity tuning index

$$
=\frac{\text { maximum response }- \text { minimum response }}{\text { maximum response }+ \text { minimum response. }}
$$

The two measures are strongly correlated $(r=0.93)$, indicating that changes in vergence over this range have little effect on disparity selectivity. So at least over the more modest range of vergence angles studied here (and most critically for the vergence range explored during the disparity-clamp experiments), the effects of vergence angle on disparity tuning are slight and should not disrupt the analysis of the disparity clamp data presented earlier.

Nonetheless, a close analysis shows that there are some links between disparity tuning and vergence state within our data set. A specific example is shown in Figure 13, which plots the disparity tuning function for one neuron recorded at two vergence angles (the same neuron as shown in Figs. 6-9). The Figure also shows the Gabor+Shift fit as presented earlier. For near fixation, the tuning curve is shifted slightly to the left (toward uncrossed disparities). This shift could not be explained easily in terms of relative disparity, but it could arise if the neuron is selective for absolute disparity and the animal is not converging accurately. Figure 13 also shows the distribution of measured fixation disparities for the two vergence conditions. The change in the mean fixation disparity between the two conditions inevitably produces a shift in the absolute disparity of the stimuli.

A shift of this kind, created by a change of fixation disparity, would appear as an interaction between vergence position and stereo disparity (as assessed in terms of the stimuli presented
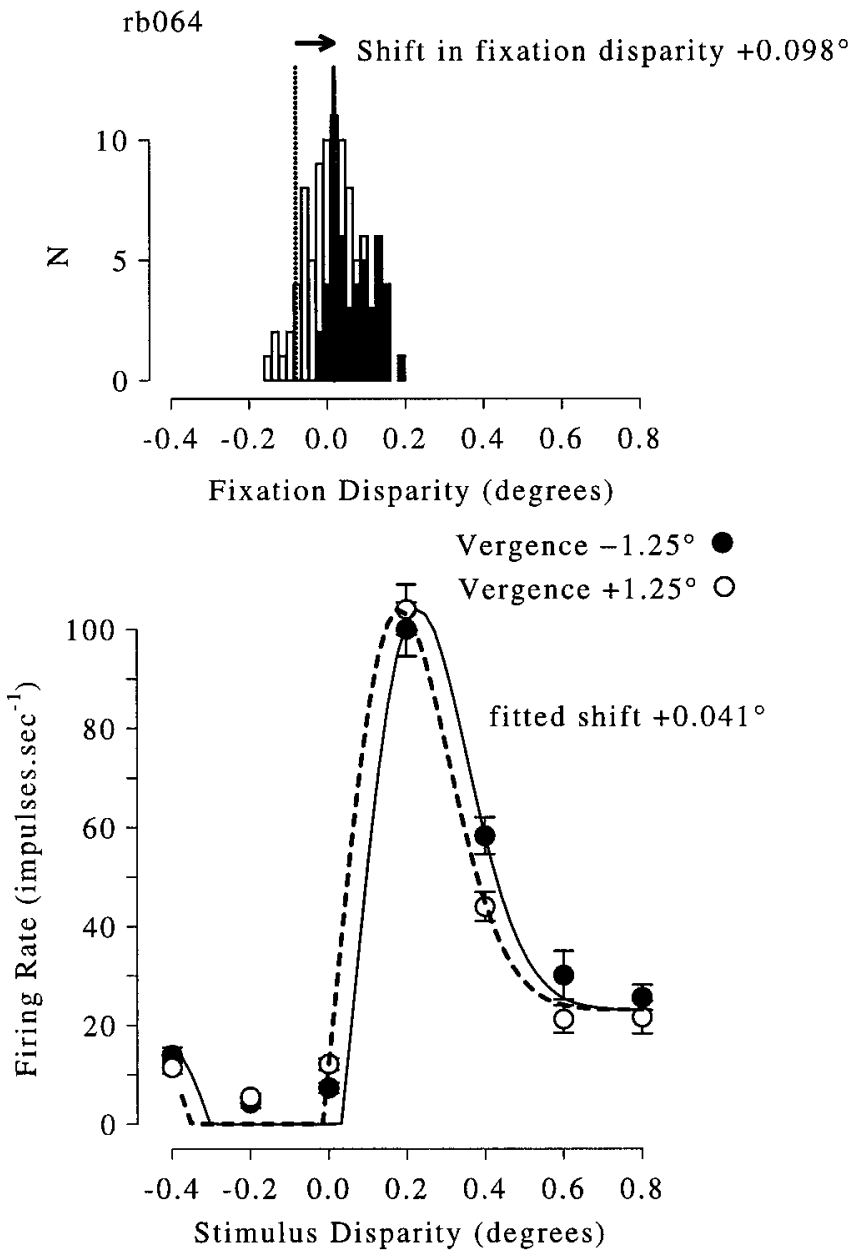

Figure 13. Effects of change in mean vergence on disparity selectivity for one neuron. The stimulus disparity plotted is the relative disparity between the foreground and the fixation marker (because there was no controlled manipulation of the absolute disparity). As in Figure 7, the absolute disparity of the fixation marker (i.e., fixation disparity) was calculated for each trial, and frequency histograms for this are shown in the top panel. There is a small difference in the mean fixation disparity at the two vergence angles, and this is reflected by a similar shift in the disparity tuning when expressed in terms of relative disparity. The direction of the shift corresponds to that predicted on the basis that the neuron is fundamentally selective for absolute disparity.

directly on the CRT screen) only if the neurons under study were primarily sensitive to absolute disparity. If the neurons were primarily sensitive to relative disparity, then there should be no change in the tuning under small fluctuations of vergence angle [as argued by Motter and Poggio (1984, 1990)]. The next section examines measures of fixation disparity across the sample of neurons that we have studied. Fixation disparities are small, hard to measure, and easily confounded with instrumental errors in the eye-position signals. Therefore ultimately we place greater reliance on the data obtained during disparity clamps. Nonetheless, the effects of fixation disparity appear to support the same conclusion.

\section{Analysis of fixation disparities}

If V1 neurons are selective for absolute disparity, then the influence of changes in the mean fixation disparity should be evident on a plot like that shown in Figure 13, which plots responses in 


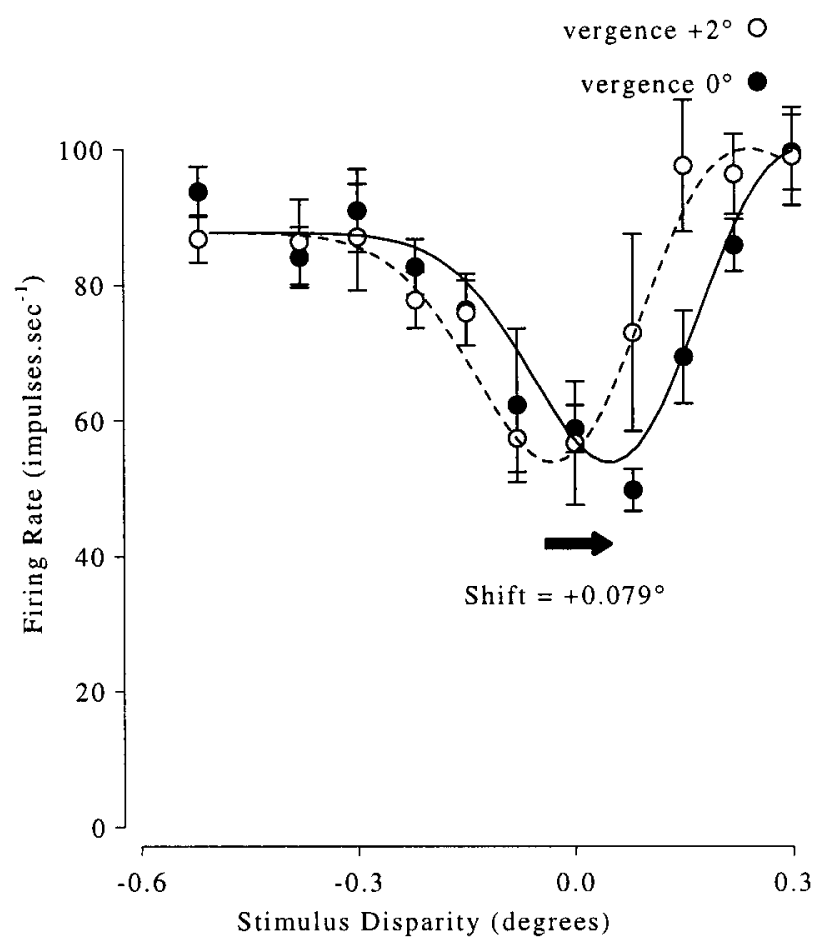

Figure 14. Effects of change in mean vergence on disparity selectivity for a neuron from monkey $\mathrm{Hg}$. In this animal, changes in the vergence stimulus induced larger changes in fixation disparity than for monkey $\mathrm{Rb}$. The relative disparity tuning curves also tended to show larger shifts, as shown here.

terms of the stimulus presented on the CRT monitors. The haploscopic presentation used here changes the stimulus to the vergence system without changing the stimulus to the accommodation system; thus, the magnitude of the vergence response is expected to be smaller than the magnitude of the vergence stimulus (Judge, 1991; Cumming and Judge, 1986; Howard and Rogers, 1995). (Note that because the eye movement signals were calibrated with conjugate movements, we do not have to make any assumptions concerning the vergence behavior of the animals to make this measurement.) Unlike the situation during disparity clamp measurements, the size of any fixation disparity now depends on the characteristics of the animal's vergence system. During the clamp, the size of the additional absolute disparity was controlled by the feedback loop.

Nonetheless, an interpretation in terms of fixation disparity makes a clear prediction about the direction of the shift in tuning: the animal should be underconverged when fixation is near, moving the plotted disparity tuning function toward uncrossed disparities. That is the direction of shift seen in Figures 13 and 14, the latter showing a larger shift from the second monkey. The shift in tuning was in the predicted direction in 47/53 cases. To assess the significance of the shift, a sequential $F$ test was performed. The fitted shift was significant $(p<0.05)$ for $42 / 53$ neurons, only one of which was in the direction opposite to that predicted by absolute disparity tuning.

Figure 15 shows the relationship between the measured change in fixation disparity and the fitted shift in disparity tuning for each neuron. The important feature of this figure is that for the great majority of neurons both the change in fixation disparity and the shift in disparity tuning have the same sign. There is also a weak, but statistically significant, correlation $(r=0.43, t$ test $0.01<p<$

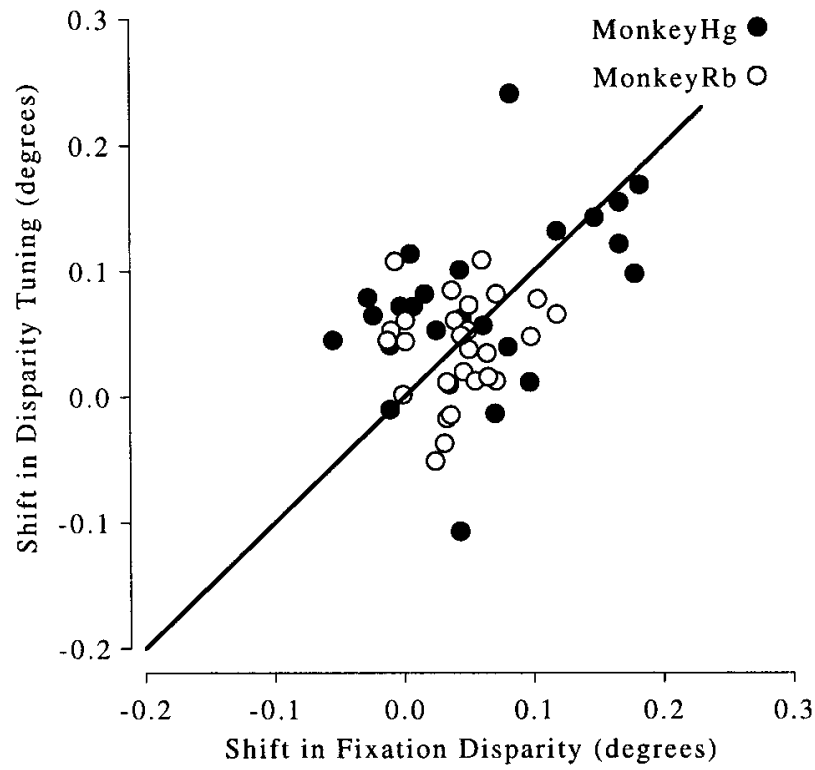

Figure 15. Scatterplot showing relationship between measured change in fixation disparity and fitted shift in disparity tuning for each cell. Most of the shifts are in the same direction (positive) for both parameters. Thus these data clearly indicate that on average the animals underconverged for near targets, and the absolute disparity that this adds to the stimuli is reflected in the cell firing. There is also a weak but significant $(0.01<p<$ $0.05)$ correlation between the two shifts. This correlation is also significant in the data for Monkey $\mathrm{Hg}$ alone, which shows a wider scatter and a larger mean change (for both fixation disparity and disparity tuning). The correlation is not significant in the data for Monkey $R b$ alone.

0.05). It is interesting to note that the shifts in the disparity tuning curves tend to be larger and more variable for monkey $\mathrm{Hg}$ (mean $\left.-0.077 \pm 0.067^{\circ} \mathrm{SD}\right)$ than for monkey $\mathrm{Rb}\left(\right.$ mean $-0.037 \pm 0.039^{\circ}$ $\mathrm{SD})$. The same pattern is evident when comparing the pattern of fitted shifts between the two animals $\left(\mathrm{Hg}-0.058 \pm 0.068^{\circ}, \mathrm{Rb}\right.$ $\left.-0.042 \pm 0.033^{\circ}\right)$, suggesting that the fixation disparity of monkey $\mathrm{Hg}$ was somewhat more variable than that of $\mathrm{Rb}$. However, the change in fixation disparity is so small $(<3 \%$ of the vergence change) that it would be unwise to place much emphasis on the measured difference in fixation disparity between the two monkeys. This analysis also indicates just how much calibration errors in the eye movement signal will affect a measure of fixation disparity. Consider the effects of a $1 \%$ error in the calibration of the eye position gain. Over the range of vergence angles used here (usually $2.5^{\circ}$ ), this would produce an apparent fixation disparity of $>0.025^{\circ}$. This again emphasizes the superiority of the results obtained using feedback. Because the mean vergence angle is similar for the two clamp conditions, small errors in calibration will not lead to large misestimates of the clamp sizes.

In summary, the disparity tuning data collected during periods of steady fixation at two different vergence angles indicate that the selectivity of the neurons for absolute disparity is the same in both conditions, but that a slight failure of the monkeys to converge accurately leads to small differences in the responses to stimuli that are identical on the CRT monitors. Together with the results from the previous section, this indicates that disparityselective neurons in V1 are primarily selective for the absolute disparity of the stimulus within their receptive field. This relationship can be summarized across all four conditions studied by superimposing absolute disparity tuning curves. This is done for four cells (two from each monkey) in Figure 16. 


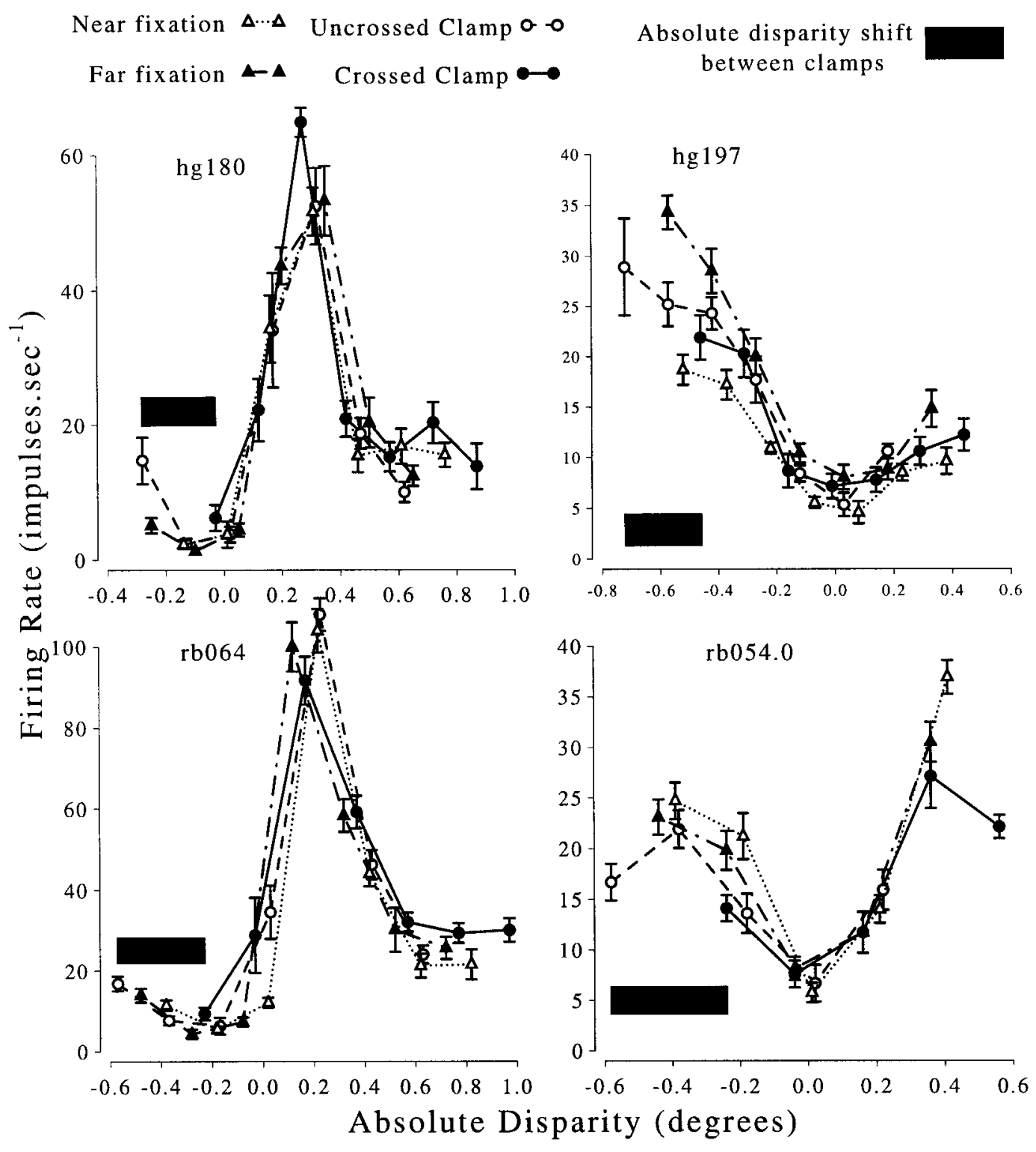

Figure 16. Summary of responses under all four conditions studied, for four different units. For each neuron, four disparity tuning functions are shown, corresponding to the four conditions shown in Figure 4. In each case, the measured disparity of the fixation marker has been added to the stimulus relative disparity, to estimate the absolute disparity of the stimulus within the receptive field. The magnitude of the shift in absolute disparity produced by the clamps is shown by the solid bars. If the neurons were selective for relative disparity, the tuning curves for the two clamp conditions should appear displaced horizontally by this distance. In both monkeys, and for all types of tuning (T0/TE, Near/Far, T1), neurons show a consistent relationship to absolute disparity.

Up to this point, we have demonstrated that the general shape of the disparity tuning function is not greatly different over the range of vergence angles that we explored in this study. We have also shown that fixation disparities produce consistent and predictable effects on the disparity tuning of V1 cortical neurons. Nonetheless, fixation disparities should create no more than a horizontal shift of the disparity tuning function along the abscissa. It was apparent in at least a few cases that a horizontal shift was insufficient to align the disparity tuning functions measured at two different static vergence positions.

A statistical comparison of the disparity tuning at two vergence positions was made by fitting the data first with the Gabor+Shift model (as in the preceding sections) and second with two independent Gabor functions. For 13/53 units, the independent Gabor functions produced a significantly better fit $(p<0.05)$ than the
Gabor + Shift fit. However, even in cases in which the model using two independent Gabor fits did produce a significantly improved fit, this typically only accounted for a relatively small fraction of the total variance within the data set (the same was true for the data presented from the clamp conditions). Across the population, the Gabor+Shift model already accounted for $89 \%$ of the variance. The use of two independent Gabor functions accounted for an additional $8 \%$, boosting the total variance explained to $97 \%$. Although this is a relatively small effect, it is a significant departure from a simple encoding of absolute disparity coupled with the expected effects of fixation disparities. Some possible causes are now considered.

Figure 17 shows data for the most extreme example we encountered, in which the Gabor+Shift fit accounts for only $41 \%$ of the variance, whereas two independent Gabors account for $97 \%$. For 
A)

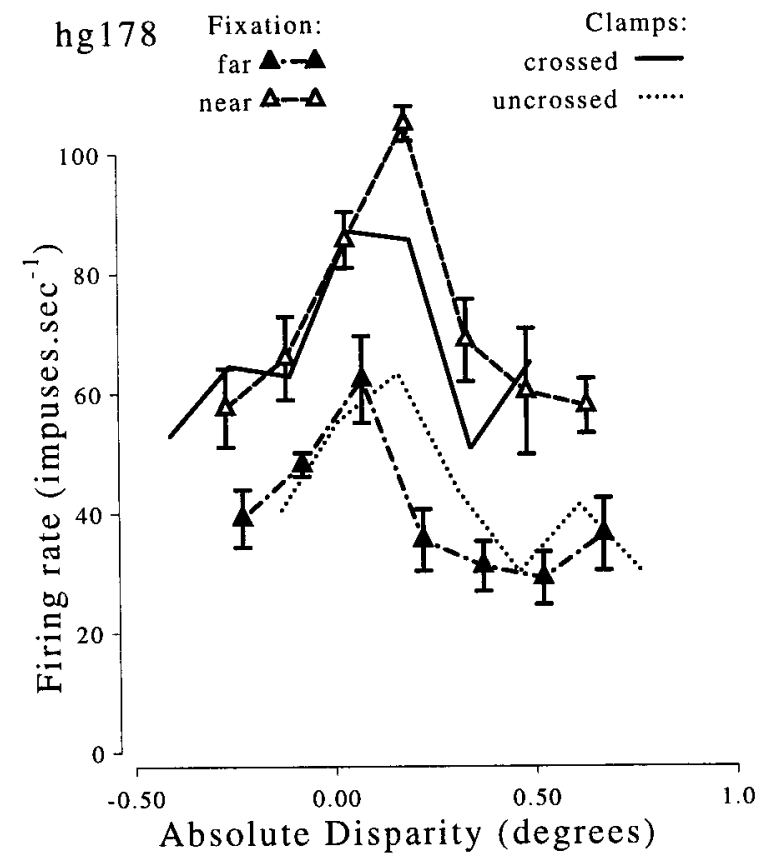

B)

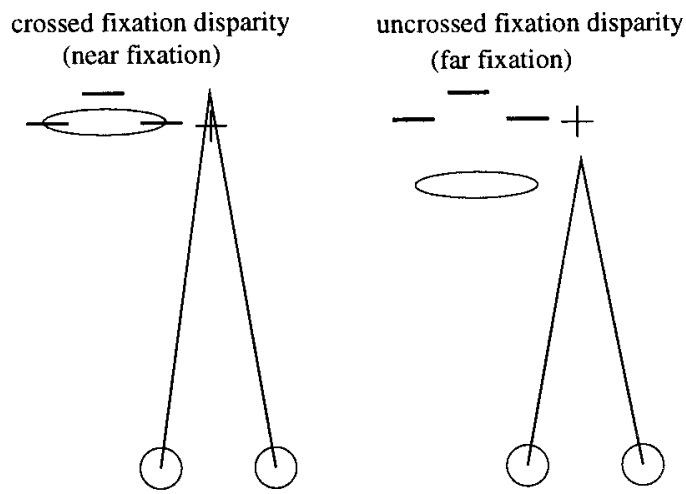

Figure 17. A, Disparity tuning of one neuron whose response rate was substantially altered by changes in vergence angle. For this reason, fitting the absolute disparity tuning data with a single Gabor gave a poor fit (the worst in our entire data set; see Fig. 10). The apparent effect of vergence could be the result of underestimating the size of the receptive field, because changes in fixation disparity produce changes in the absolute disparity of the surround region of the stimulus $(B)$. If this surround region were to encroach on the receptive field, then the change in fixation disparity would alter the absolute disparity of stimuli within the RF and hence alter neuronal firing. Note that the direction of the change in firing rate fits with this explanation. The neuron is tuned to small crossed disparities, which is the type of fixation disparity produced by near fixation (during which firing is greater). This explanation is also supported by the fact that the tuning during uncrossed clamps closely resembled that during far fixation. Similarly, tuning during crossed clamps resembled that during near fixation. all but four neurons (two from each animal) the Gabor + Shift fit accounted for $>70 \%$ of the variance, so this example is truly extreme. However, among those neurons where the fit with independent Gabor functions was significantly better, the pattern of results was common: there was a change in the mean firing rate between the two vergence states. Among those cells that showed a statistically identifiable change in the disparity tuning function, the change was always well described by a change in either mean firing rate or amplitude of the Gabor (sequential $F$ test: $p<0.05$ for at least one of these tests in all 13 cells).

This phenomenon resembles that described by Trotter et al. (1997): changes in vergence angle are associated with changes in the modulation of firing rates caused by disparity changes. However, we observed similar changes when comparing responses to the two clamp conditions, although the mean vergence angle across the two clamp conditions was very similar. An unexpected feature of the data in Figure 17 suggests an alternative explanation. The near fixation trials, associated with higher firing rates, have crossed fixation disparities and hence crossed disparities of the background. If the receptive field size had been underestimated, this crossed absolute disparity would then encroach on the receptive field and so influence activity directly. For the neuron shown in Figure 17, which is activated by crossed disparities more than uncrossed disparities, this would produce an increase in firing rate when the background had a crossed disparity. Conversely, during far fixation when the absolute disparity of the surround region is uncrossed, there would be a reduction in firing rate.

A similar pattern can be seen in the tuning curves for unit hg197 shown in Figure 16. The condition associated with stronger firing is the one in which the disparity of the background is nearer to the cell's preferred disparity. Because the number of cells that show any change in tuning between conditions is small (13) and the effects are usually much smaller than those illustrated in Figure 17, it is hard to establish in a quantitative way whether this explanation is satisfactory in every case.

\section{DISCUSSION}

The feedback method of Rashbass and Westheimer (1961) and Cumming and Judge (1986) was used to present binocular stimuli in which the absolute disparity of an entire display, including the visible fixation marker, was manipulated independently of the relative disparities between all of the visible features. This experimental procedure ensures a clear distinction between relative and absolute disparities in the stimulus and successfully produced an equally clear distinction in the neuronal responses: the firing of all 53 cells bore a more consistent relationship to absolute than to relative disparity. Indeed, for 39 of the 53 cells, there was no significant deviation from a consistent relationship between firing rate and absolute disparity. By comparison, no cells encountered in $\mathrm{V} 1$ had a consistent relationship with relative disparity. Because psychophysical evidence suggests that relative disparities are calculated over distances considerably larger than the width of V1 receptive fields (Tyler, 1973, 1974; Westheimer, 1979; McKee et al., 1990), it seems that the signals used for these psychophysical judgements are not constructed in V1.

This result may seem unsurprising "given the generally accepted notion that most neurons in V1 act as localized filters" (Ohzawa, 1998). This notion has gained acceptance, particularly from work with anesthetized animals, because so many properties can be explained on this basis. In reality even with anesthetized animals there is ample evidence for more complex responses that 
are generated by influences from outside the minimum response field (Maffei and Fiorentini, 1976; Gilbert and Wiesel, 1990; Sillito et al., 1995; Levitt and Lund, 1997). Our experiments suggest that these responses from outside the minimum response field are not used to represent relative disparities.

To turn specifically to stereo disparity and experiments with awake animals, there are several claims that stimuli outside the receptive field (RF) influence the response to disparities presented within the RF. Motter and Poggio $(1984,1990)$ found that neuronal receptive fields in V1 were less sensitive than expected to vergence movements of the eyes. This is equivalent to a degree of selectivity for relative disparity. Zipser et al. (1996) reported that changes in the disparity of stimuli outside the RF influenced responses to stimuli within the RF. In the disparity domain there is a clear functional significance that could be attributed to such interactions: measures of disparity differences between image features are essential for the best stereoacuities (Westheimer, 1979). Despite these earlier studies, which can all be interpreted as indicating a representation of relative disparity in primate V1, the data presented here clearly contradict those claims. The apparent discrepancies with earlier reports therefore require close scrutiny.

\section{Fixation disparities}

The conclusions of Motter and Poggio (1984) were all based on examining the relationship between changes in fixation disparity and disparity selectivity. This strategy places heavy reliance on the accuracy of the eye position recording. (Recall that one of the strengths of the feedback loop is that it provides an additional check on the calibration of vergence signals. See Materials and Methods.) Motter and Poggio (1984) provide little discussion of the possibility that some variability in their records of vergence may be instrumental in origin. A comparison with the sizes of fixation disparity and vergence variability in human studies indicates that this possibility deserves serious consideration.

Fixation disparities in normal human observers are usually small (Riggs and Neill, 1960; Ogle, 1964). However, even a large fixation disparity will not compromise the disparity selectivity of an absolute disparity detector, provided that the fixation disparity is stable over time. The key question is whether the variation in fixation disparity reported in humans is comparable with that reported by Motter and Poggio (1984), who reported SDs of $>7$ arcmin.

A few studies have measured precisely the position of both eyes objectively in human subjects, and they all report SDs of 1-2 arcmin, with no subject showing a SD $>3$ arcmin (St. Cyr and Fender, 1969; Collewijn et al., 1988; Enright, 1991). More recently, a forced-choice psychophysical procedure using Nonius lines has been used to place an upper limit on the variability of fixation disparities, reporting values of 1-3 arcmin (Duwaer, 1983; McKee and Levi, 1987; Jaschinski-Kruza and SchubertAlshuth, 1992). Thus human studies suggest that vergence during steady fixation is very stable. Because the variability reported by Motter and Poggio (1984) was at least fourfold greater, it seems likely that some of the large offsets reported by them were instrumental in origin. If those records of fixation disparity did have significant instrumental variation, there would be no contradiction between the results of Motter and Poggio $(1984,1990)$ and the results presented above. Our conclusion (for binocular eye movements) is similar to that reached by Gur and Snodderly (1987, 1997) concerning conjugate eye movements and receptive field location.

\section{Absolute disparity and binocular eye position}

If disparity-selective neurons in V1 are sensitive only to absolute disparity, then careful monitoring of binocular eye position is essential for interpretation of their responses, especially in the awake animal. Yet many studies on the binocular properties of V1 neurons in the awake animal have failed to record the positions of both eyes (Trotter et al., 1992, 1997; Zipser et al., 1996). Trotter et al. $(1992,1997)$ have reported changes in disparity tuning with changes in fixation distance. If the animals' fixation disparity had been different at the different viewing distances, as typically occurs (Judge, 1991), then the absolute disparity of the stimuli would actually have varied as a function of viewing distance. This will inevitably alter the neuronal response of an absolute disparity detector at different distances. Indeed this can give rise to an apparent change in modulation strength in narrowly tuned cells. This possibility is illustrated by the cell in Figure 8 (top left). The tuning curve shown with solid symbols has a larger modulation depth than that shown with open symbols, but when the two curves are shown together in terms of absolute disparity, it is clear that they are compatible with a single function whose peak is near $0^{\circ}$. The tuning curve shown with open symbols shows less modulation simply because there is no sample close enough to the true peak. Even if the mean fixation disparity were constant at different viewing distances, changes in variability of vergence at different viewing distances could also cause changes in the measured disparity tuning curves.

Only a small proportion of the neurons we studied (taking into account fixation disparities) showed any changes in disparity selectivity with vergence angle, and none showed effects as dramatic as those illustrated by Trotter et al. (1992, 1997). On the other hand, the changes in vergence angle used by Trotter et al. (viewing distances from 80 to $20 \mathrm{~cm}$, corresponding to vergence angles of $2-10^{\circ}$ for a monkey with an interocular separation of 3.5 $\mathrm{cm})$ were larger than used here $\left(1.0-3.5^{\circ}\right)$. In their work, the most dramatic effects seemed to occur at the nearest viewing distance. It is therefore possible that if we had explored larger vergence angles we would have seen similar effects. Use of large changes in the vergence stimulus also increases the likelihood of substantial changes in fixation disparity, so the possibility that the effects observed by Trotter et al. $(1992,1997)$ merely reflect changes in the absolute disparity of the stimulus cannot be discounted at this stage.

Finally, it is worth recalling that the disparity-driven vergence system requires a pure measure of absolute disparity to sustain vergence control mechanisms (Rashbass and Westheimer, 1961). If neurons in V1 signal absolute disparity in the way we have proposed here and these same neurons send a signal to the vergence system, then it could be disadvantageous to incorporate a vergence signal within the firing patterns of these neurons.

\section{Disparity-specific modulation from outside the classic receptive field}

Zipser et al. (1996) argued that the responses of V1 neurons receive a long-latency modulatory influence that is sensitive to the disparity of stimuli placed outside the classic receptive field. They attributed this to a generalized perceptual influence on V1 neurons. Their findings were not accompanied by any measurements of vergence eye movements. It is therefore quite possible that some of the effects reported by Zipser et al. (1996) are the result of vergence changes induced by manipulations of the stimulus outside each unit's RF. Clearly, studies that record vergence position are required to resolve this issue (Cumming and Parker, 
1998a,b). It is worth noting that the present experimental data also provide a test of whether there is a significant modulatory effect of surround disparities, at least for the disparity-tuned neurons investigated here. As illustrated in Figure 3, stimuli with the same absolute disparity in the receptive field, but different disparities of the fixation marker, have different cyclopean configurations. Because these different configurations produce the same neuronal response, this indicates that there is no effective modulation produced by the disparity of the surround. One important difference between the conditions used here and those of Zipser et al. (1996) is the fact that the random dot stereograms used here were dynamically refreshed over time (see Materials and Methods). Our recent evidence suggests that the static random dot stereograms used by Zipser et al. (1996) are more vulnerable to the effects of eye movements (Cumming and Parker, 1998a,b).

\section{Stereoscopic depth perception}

The present results have implications for the role played by disparity-tuned V1 cortical neurons in the perception of stereopsis. It seems that further processing of their signals, presumably outside V1, is necessary for the perception of depth. Consider the experiments of Westheimer (1979). For disparities $<1$ arcmin, subjects could only detect the depth of single line targets when an additional reference marker was present; in other words, only relative disparities were detectable. It may be that even without the reference marker, the activity of V1 neurons reliably signals the change in disparity of the target, but the subject cannot determine whether this change in neural activity results from a target displacement or a change in vergence eye position. Relative disparity signals are required to make this distinction, but our results suggest that these relative disparities are not explicitly represented in V1. This implies that the signals used for depth detection in this task come from some other brain area. Of course, the addition of a reference marker does change the activity of other neurons in V1 (i.e., those neurons for which the reference marker lies within their receptive field), so that information about relative disparity is implicitly available from the entire population of V1 neurons. However, no simple pooling of responses from neurons in one part of the visual field will explain the psychophysical response. If cells outside V1 received input from disparity-selective neurons in different parts of the visual field and extracted a difference signal (Westheimer, 1979), they might account for the observed psychophysical performance.

When thinking about physiological responses to the bar stimuli used by Westheimer (1979), it seems that the perception of depth of a test bar is altered by a manipulation (the addition of a reference mark) that does not influence the response of V1 neurons to the test bar itself. The opposite effect is found for wide-field displays. Here changes in the absolute disparity should produce substantial changes in the activity of many disparityselective neurons in V1, and yet this produces no change in the sensation of depth (Regan et al., 1986). Once again, if cells in some other brain area calculated difference signals based on the activity of disparity-selective V1 neurons, activity in such cells would more closely match the perception of depth in this stimulus. We have argued previously that processing beyond V1 is required to account for how image features are matched between the two eyes (Cumming and Parker, 1997). Our current view is that disparity selectivity in primate $\mathrm{V} 1$ is only a preliminary stage in stereopsis, so that the further processing outside V1 (most likely using signals from disparity-selective neurons in V1) is responsible for generating the sensation of depth.

\section{REFERENCES}

Barlow H, Blakemore C, Pettigrew J (1967) The neural mechanisms of binocular depth discrimination. J Physiol 193:327-342.

Bishop PO, Henry GH (1971) Spatial vision. Annu Rev Psychol 22:119-160.

Brookes A, Stevens KA (1989) The analogy between stereo depth and brightness. Perception 18:601-614.

Collewijn H, Erkelens C, Steinman R (1988) Binocular co-ordination of human horizontal saccadic eye movements. J Physiol (Lond) 404:157-182.

Cumming BG, Judge SJ (1986) Disparity-induced and blur-induced convergence eye movement and accommodation in the monkey. J Neurophysiol 55:896-914.

Cumming BG, Parker AJ (1997) Responses of primary visual cortical neurons to binocular disparity without the perception of depth. Nature 389:280-283.

Cumming BG, Parker AJ (1998a) Eye movements explain contextual modulation by disparity in primate V1. Soc Neurosci Abstr 24:1981.

Cumming BG, Parker AJ (1998b) Vergence eye movements explain some aspects of contextual modulation in primate V1. Perception 27:20.

Draper NR, Smith H (1966) Advanced regression analysis. New York: Wiley.

Duwaer AL (1983) New measures of fixation disparity in the diagnosis of binocular oculomotor deficiences. Am J Optom Physiol Opt 60:586-597.

Enright J (1991) Exploring the third dimension with eye movements: better than stereopsis. Vision Res 31:1549-1546.

Erkelens CJ, Collewijn H (1985) Motion perception during dichoptic viewing of moving random-dot stereograms. Vision Res 25:583-588.

Gilbert CD, Wiesel TN (1990) The influence of contextual stimuli on the orientation selectivity of cells in primary visual cortex of the cat. Vision Res 30:1689-1701.

Gur M, Snodderly D (1987) Studying striate cortex neurons in behaving monkeys: benefits of image stabilization. Vision Res 27:2081-2087.

Gur M, Snodderly D (1997) Visual receptive fields of neurons in primary visual cortex (V1) move in space with the eye movements of fixation. Vision Res 37:257-265.

Howard IP, Rogers BJ (1995) Binocular vision and stereopsis. Oxford: OUP.

Jaschinski-Kruza W, Schubert-Alshuth E (1992) Variability of fixation disparity and accommodation when viewing a CRT visual display unit. Ophthal Physiol Opt 12:411-419.

Joshua DE, Bishop PO (1970) Binocular single vision and depth discrimination. Receptive field disparities for central and peripheral vision and binocular interaction on peripheral single units in cat striate cortex. Exp Brain Res 10:389-416.

Judge SJ (1991) Vergence. In: Vision and visual dysfunction (Carpenter R, ed), pp 157-172. London: Macmillan.

Judge SJ, Richmond BJ, Chu FC (1980) Implantation of magnetic search coils for measurement of eye position: an improved method. Vision Res 30:535-538.

Levitt JB, Lund JS (1997) Contrast dependence of contextual effects in primate visual cortex. Nature 387:73-76.

Maffei L, Fiorentini A (1976) The unresponsive regions of visual cortical receptive fields. Vision Res 1131-1139.

McKee SP, Levi DM (1987) Dichoptic hyperacuity: the precision of nonius alignment. J Opt Soc Am 4:1104-1108.

McKee SP, Welch L, Taylor DG, Browne SF (1990) Finding the common bond: stereoacuity and other hyperacuities. Vision Res 30:879-891.

Merrill EG, Ainsworth A (1972) Glass-coated platinum-plated tungsten electrodes. Med Biol Eng 10:662-672.

Motter BC, Poggio GF (1984) Binocular fixation in the rhesus monkey: spatial and temporal characteristics. Exp Brain Res 54:304-314.

Motter BC, Poggio GF (1990) Dynamic stabilization of receptive fields of cortical neurons (V1) during fixation of gaze in the macaque. Exp Brain Res 83:37-43.

Mountcastle VB, Lynch JC, Georgopoulos A, Sakata H, Acuna C (1975) Command functions for operations within extrapersonal space. J Neurophysiol 38:871-908. 
Nikara T, Bishop PO, Pettigrew JD (1968) Analysis of retinal correspondence by studying receptive fields of binocular single units in cat striate cortex. Exp Brain Res 6:353-372.

Ogle KN (1964) Researches in binocular vision. New York: Hafner.

Ohzawa I (1998) Mechanisms of stereoscopic vision: the disparity energy model. Curr Opin Biol 8:509-515.

Ohzawa I, DeAngelis GC, Freeman RD (1990) Stereoscopic depth discrimination in the visual cortex: neurons ideally suited as disparity detectors. Science 249:1037-1041.

Poggio G (1995) Mechanisms of stereopsis in monkey visual cortex. Cereb Cortex 3:193-204.

Poggio GF, Fisher B (1977) Binocular interactions and depth sensitivity in striate and prestriate cortex of behaving rhesus monkey. J Neurophysiol 40:1392-1405.

Poggio GF, Talbot WH (1981) Mechanisms of static and dynamic stereopsis in foveal cortex of the rhesus monkey. J Physiol (Lond) 315:469-492.

Rashbass C, Westheimer G (1961) Disjunctive eye movements. J Physiol (Lond) 159:326-338.

Regan D, Erkelens CJ, Collewijn H (1986) Necessary conditions for the perception of motion-in-depth. Invest Ophthalmol Vis Sci 27:584-597.

Riggs LA, Neill EW (1960) Eye movements recorded during convergence and divergence. J Opt Soc Am 50:913-920.

Rogers BJ, Graham ME (1982) Similarities between motion parallax and stereopsis in human depth perception. Vision Res 22:261-270.

Sillito AM, Grieve KL, Jones HE, Cudeiro J, Davies J (1995) Visual cortical mechanisms detecting focal orientation discontinuities. Nature 378:492-496.

Skottun B, DeValois R, Grosof D, Movshon J, Albrecht D, Bonds A (1991) Classifying simple and complex cells on the basis of response modulation. Vision Res 31:1079-1086.

St. Cyr GF, Fender DH (1969) The interplay of drifts and flicks in binocular fixation. Vision Res 9:245-265.

Trotter Y, Celebrini S, Stricanne B, Thorpe S, Imbert M (1992) Modulation of neural stereoscopic processing in primate area-V1 by the viewing distance. Science 257:1279-1281.

Trotter Y, Celebrini S, Stricanne B, Thorpe S, Imbert M (1997) Neural processing of stereopsis as a function of viewing distance in primate visual cortical area V1. J Neurophysiol 76:2872-2885.

Tyler CW (1973) Stereoscopic vision: cortical limitations and a disparity scaling effect. Science 181:276-278.

Tyler CW (1974) Depth perception in disparity gratings. Nature 251:140-142.

Westheimer G (1979) Cooperative neural processes involved in stereoscopic acuity. Exp Brain Res 36:585-597.

Wheatstone C (1838) Contributions to the physiology of vision I: on some remarkable, and hitherto unobserved, phenomena of vision. Philos Trans R Soc B Biol Sci 13:371-395.

Wurtz R (1969) Visual receptive fields of striate cortex neurons in awake monkeys. J Neurophysiol 36:727-242.

Zipser K, Lamme VF, Schiller PH (1996) Contextual modulation in primary visual cortex. J Neurosci 16:7376-7389. 